\title{
Mechanisms Underlying Spatial Representation Revealed through Studies of Hemispatial Neglect
}

\author{
Marlene Behrmann ${ }^{1}$, Thea Ghiselli-Crippa ${ }^{2}$, John A. Sweeney ${ }^{2}$, \\ Ilaria Di Matteo ${ }^{1}$, and Robert Kass ${ }^{1}$
}

\begin{abstract}
The representations that mediate the coding of spatial position were examined by comparing the behavior of patients with left hemispatial neglect with that of nonneurological control subjects. To determine the spatial coordinate system(s) used to define "left" and "right," eye movements were measured for targets that appeared at $5^{\circ}, 10^{\circ}$, and $15^{\circ}$ to the relative left or right defined with respect to the midline of the eyes, head, or midsaggital plane of the trunk. In the baseline condition, in which the various egocentric midlines were all aligned with the environmental midline, patients were disproportionately slower at initiating saccades to left than right targets, relative to the controls. When either the trunk or the head was rotated and the midline aligned with the most
\end{abstract}

peripheral position while the eyes remained aligned with the midline of the environment, the results did not differ from the baseline condition. However, when the eyes were rotated and the midline aligned with the peripheral position, saccadic reaction time (SRT) differed significantly from the baseline, especially when the eyes were rotated to the right. These findings suggest that target position is coded relative to the current position of gaze (oculocentrically) and that this eyecentered coding is modulated by orbital position (eye-in-head signal). The findings dovetail well with results from existing neurophysiological studies and shed further light on the spatial representations mediated by the human parietal cortex.

\section{INTRODUCTION}

Reaching to pick up a cup requires that the spatial position of the cup is rapidly and accurately represented. The process of spatial representation, however, is fraught with problems. The spatial location of the cup is initially registered with respect to the coordinates of the retina, but the reach is executed to a position defined relative to the acting limb. Moreover, there are inhomogeneities in the receptor surfaces of the different modalities such that, in vision, a disproportionately large region of the primary visual cortex represents information that appears foveally, whereas, in the motor cortex, there is overrepresentation of regions mediating fine movements (Stein, 1992). How spatial position, defined in one set of coordinates, is translated to another has been the subject of numerous investigations, from neurophysiological studies with nonhuman primates to human functional imaging studies, but, despite this, the coordinate transformation process remains poorly understood.

Existing data from studies with nonhuman primates have suggested that the posterior parietal cortex represents spatial information with respect to many, different frames of reference (Colby, 1998). Moreover, information from various sensory modalities may be combined

${ }^{1}$ Carnegie Mellon University, ${ }^{2}$ University of Pittsburgh

in order to derive more complex and increasingly abstract representations of space (Andersen, Snyder, Li, \& Stricanne, 1993; Andersen, 1995; Andersen, Snyder, Bradley, \& Xing, 1997). For example, in addition to spatial position being mapped with respect to the retina or current position of gaze, namely, oculocentrically (Colby, Duhamel, \& Goldberg, 1995), information about the spatial position of an object imaged on the retina may be combined with the (extraretinal) position of the eyes in the orbit to provide a mapping of the position of an object with respect to the head (head-centered coordinates). Furthermore, combining the eye and head position information with neck proprioception or efference copy from neck muscles (head with respect to body) enables spatial position to be defined with reference to the body. Finally, combining the eye and head position signals with vestibular signals enables the derivation of a more abstract and general reference frame centered on the world (Snyder, Grieve, Brotchie, \& Andersen, 1998) (although see Colby, 1998 for a somewhat different view). On these accounts, the parietal cortex plays a critical role in representing spatial information and in transforming sensory input to an actionbased code. Downstream areas concerned with motor planning and execution, such as frontal and premotor areas, can then access these different spatial representations selectively for the purpose of action. 
There are several important findings from these studies. The first is that information in the parietal cortex is represented with respect to its position on the retina. Thus, neurons in this region (specifically, in the lateral intraparietal region (LIP) and BA 7a; Colby et al., 1995; Colby \& Goldberg, 1999) carry signals that describe stimuli in terms of their direction and distance relative to the center of gaze. Moreover, neurons in this area update the internal representation of space in conjunction with eye movements so that the representation always matches the current eye position, thereby maintaining eye-centered coordinates (Colby et al., 1995; Colby, \& Goldberg, 1999; Duhamel, Colby, \& Goldberg, 1992). Interestingly and counterintuitively, spatial position is coded relative to retinal coordinates even when the input is not visual; for example, when monkeys make delayed saccades to auditory signals, neurons in LIP code the location of the stimulus in eye-centered coordinates (Stricanne, Andersen, \& Mazzoni, 1996). Finally, representations derived for action also appear to be coded with respect to the retina; during reaching, the neuronal response in the posterior reach region (PRR) of the parietal cortex is sensitive to the retinal location of the target but not the starting point of the reach (Batista, Buneo, Snyder, \& Andersen, 1999; see also DeSouza et al., 2000; Pouget, Ducom, Torri, \& Bavelier, 2001). These findings all attest to the central role of retinocentric coordinates in spatial representation. The first goal of this article is to examine the evidence for eye-centered coding of spatial information in the human parietal cortex.

A second important conclusion from the existing studies is that various inputs may be combined to yield different spatial reference frames. This is probably best illustrated by the finding that the response amplitude of the retinotopically mapped parietal neurons may be modulated by eye position (Andersen, Essick, \& Siegel, 1985; see also DeSouza et al., 2000). This convergence produces cells with retinal receptive fields that are modulated in a monotonic fashion by the orbital position of the eyes and, across a population of cells with different eye and retinal position sensitivities, yields a unique pattern of firing depicting location in head-centered coordinates (Xing \& Andersen, 2000a,b; Mazzoni, Andersen, \& Jordan, 1991; Zipser \& Andersen, 1988). Both the retinocentric coding and the multiplicative effects of retinal position combined with orbital position have been simulated in a neural network model that combines sensory and postural signals to give rise to multiple frames of reference (Pouget \& Snyder, 2000; Pouget \& Sejnowski, 1997a,b,c, 1999, 2001). The simulations involve units that compute basis functions of sensory inputs by multiplying the responses of parietal cells, characterized as a Gaussian function of retinal location, with a sigmoid function depicting eye position. A second goal of the current article, then, is to examine whether, in the parietal cortex in humans, there is also concrete evidence for a spatial representation that combines retinal and orbital eye position.

The approach that we adopt is to examine the behavior of humans who, after acquired brain damage, exhibit a disorder known as hemispatial neglect (see Bartolomeo \& Chokron, 2001; Bisiach \& Vallar, 2000). Following a right parietal lobe lesion, for example, these patients typically draw features only on the right of a picture and reach or direct their gaze more often to the right than the left. They may also shave or apply make-up only to the ipsilateral side and, finally, may show neglect of visual, auditory, and tactile stimuli. Importantly, the failure to process contralateral information is not attributable to a primary sensory or motor problem. Rather, neglect is thought to occur because neurons in one hemisphere have predominant, although not exclusive representation of the contralateral side of space; removing neurons therefore impairs, to a greater extent, spatial representations for contralateral than for ipsilateral positions (Pouget \& Driver, 2000; Rizzolatti, Berti, \& Gallese, 2000).

Hemispatial neglect is observed with greater frequency and severity after right than left lesions and, thus, we refer to neglect as left-sided. The logic of this study is to identify what "left" refers to. Put simply, when these patients ignore information on the left, what is it "left" of? Because spatial position cannot be coded absolutely but only with respect to a set of coordinates, determining what the midline is such that information to its left is ignored will elucidate the nature of the reference frames mediated by the human parietal cortex.

\section{Relevant Neuropsychological Data}

The clearest result from studies in neglect patients is that spatial position is coded in multiple reference frames. Thus, patients neglect information on the left defined egocentrically (centered on the head and/or trunk) and/or allocentrically (centered on the environment and/or object in the scene) (e.g., Hillis \& Rapp, 1998; Beschin, Cubelli, Della Sala, \& Spinazzola, 1997; Behrmann \& Moscovitch, 1994; Behrmann \& Tipper, 1999; Behrmann, 2000; Moscovitch \& Behrmann, 1994; Karnath, Schenkel, \& Fisher, 1991; Karnath \& Ferber, 1999; Farah, Brunn, Wong, Wallace, \& Carpenter, 1990; Calvanio, Petrone, \& Levine, 1987; Làdavas, 1987; Làdavas, Pesce, \& Provinciali, 1989).

Despite the plethora of studies and evidence for multiple spatial representations, evidence for coding of position with respect to the retina and current direction of gaze is less well established. Suggestive evidence from visual search studies is consistent with this account, however, as patients typically show a linear increase in fixations and their duration on the right compared to the left (Behrmann, Barton, Watt, \& Black, 1997; Hornak, 1992; Karnath \& Huber, 1992; Karnath \& Fetter, 1995; Karnath, Fetter, \& Dichgans, 1996). There is also 
some, albeit scant, evidence for modulation by eye position. For example, Kooistra and Heilman (1989) described a patient who appeared to have a left hemianopia when the eyes were fixated ahead but who showed no deficit when the eyes were deviated to the right. Because the so-called hemianopia abated when the eyes were directed rightwards, the deficit was interpreted as one of neglect rather than hemianopia (Vuilleumier, Valenza, Mayer, Perrig, \& Landis, 1999; Nadeau \& Heilman, 1991; Rapscak, Watson, \& Heilman, 1987).

The purpose of the present study, then, is to explore further the influence of the retinocentric axis and the position of the eyes on the pattern of neglect. The paradigm adopted requires subjects to saccade to targets that appear in different regions of space. Because it has been suggested that the ability to attend to various locations in space depends on brain areas that are involved in organizing goal-directed actions to them (Colby, 1998; Snyder, Batista, \& Andersen, 1997; Rizzolatti \& Camarda, 1987), we expect to observe a robust influence of retinocentric coding and gaze position on eye movements. Whereas we do not always see effects of eye position in tasks that require subjects to bisect a line or to read text (Vuilleumier et al., 1999; Schindler \& Kerkhoff, 1997), we expect to see eye position effects when subjects have to plan and execute saccades.

Eye movements have been used successfully to describe the behavior of neglect patients (e.g., Barton, Behrmann, \& Black, 1998; Behrmann et al., 1997; Gainotti, 1993). Although patients with parietal lesions typically do not have a fundamental oculomotor deficit (Niemeier \& Karnath, 2000; Gainotti, 1993; Walker, Findlay, Young, \& Welch, 1991; Chedru, Leblanc, \& Lhermitte, 1973), they do show "neglect" in their eye movements, making few contralesional saccades and showing a delay in the planning of those saccades (Braun, Weber, Mergner, \& Schulte-Monting, 1992; Johnston, 1988; Girotti, Casazza, Musicco, \& Avanzini, 1983). The impairment in contralesional saccades is not attributable to a hemianopia (Behrmann et al., 1997; Zihl, 1995; Meienberg, Zangemeister, Rosenberg, Hoyt, \& Stark, 1981) but, instead, is thought to reflect the impaired reflexive exploration of contralesional visual space and the subsequent failure to direct oculomotor action to that side (Heide \& Kömpf, 1998). The critical question is, when patients with neglect make fewer and briefer saccades to the left, with respect to what coordinate(s) are these eye movements calibrated?

In this study, the subject faces an arc of light-emitting diodes (LEDs), in which one LED is illuminated and fixated. Following varying temporal intervals, a second LED is illuminated and subjects saccade to this target. We measure the delay and accuracy with which an eye movement is initiated. This "overlap" procedure, in which the target and fixation LED appear concurrently for some amount of time, is especially sensitive to the presence of neglect (Heide \& Kömpf, 1998). The meth- od is schematically depicted in Figure 1, which shows the subject seated in the array with the seven critical LEDs included. In the baseline condition (a), the midlines of the subject's eyes, head, and trunk are centered on environmental (or world) midline, and we expect that targets on the left will be poorly acquired. Because all these various reference frames are aligned, however, we do not know whether the left-right asymmetry arises because the spatial positions are located on the left of the eyes, or of the head, or of the trunk and/or of the environment.

To determine the individual contribution of the different egocentric reference frames, we orthogonally rotate the midline of the eyes, head, or trunk out of alignment of the other frames and then examine the eye movements to targets appearing on their relative left or right as follows (for similar approach, see Karnath et al., 1991; Karnath, Christ, \& Hartje, 1993): (a) baseline (B); (b) head left (HL; Figure 1b); (c) head right (HR; Figure 1e); (d) trunk left (TL; Figure 1c), (e) trunk right (TR; Figure 1f); (f) eyes left (EL: Figure 1d); and (g) eyes right (ER; Figure $1 \mathrm{~g}$ ). In the first five conditions (a-e), subjects saccade to targets at $5^{\circ}, 10^{\circ}$, and $15^{\circ}$ to the left and right of the environment (or of fixation, as the eyes are aligned with $0^{\circ}$ environment). In the final two conditions, because the eyes are deviated away from $0^{\circ}$, the targets are located at $-10^{\circ},-5^{\circ}, 0^{\circ}, 5^{\circ}, 10^{\circ}$, and $15^{\circ}$ in the EL condition, and at $10^{\circ}, 5^{\circ}, 0^{\circ},-5^{\circ},-10^{\circ}$, and $-15^{\circ}$ in the ER condition, defined with respect to the environment. To determine whether "left" is defined with respect to one of these egocentric reference frames, we compare the behavior of the subjects in the rotation conditions, relative to the baseline, for targets that occupy the same retinal distance. We measure both intercept and slope differences in saccadic reaction time (SRT) and accuracy for left and right targets, as a function of target eccentricity since neglect generally increases with more contralesional targets (Cate \& Behrmann (submitted); Kinsbourne, 1994).

To make our predictions explicit, we present hypothetical data that would support the claim that eye movements are planned to spatial positions defined retinocentrically. If spatial position were defined only with respect to the retinal midline (see Figure 2a), then the sole determinant of performance would be the position of targets relative to the retinal axis. When the eyes are straight ahead, as in the B, HL, HR, TL, and TR conditions, left targets would be more poorly acquired than those on the right, and there would be no difference among the various other conditions. The same pattern would be obtained when the eyes are rotated, with targets to the left of fixation being acquired more poorly than targets to the right, independent of orbital position. Figure $2 \mathrm{~b}$ illustrates the further prediction that, in addition to retinocentric coding, performance may be modulated by the eye-in-head signal. As before, left targets in B, HL, HR, TL, and TR, would be more poorly 


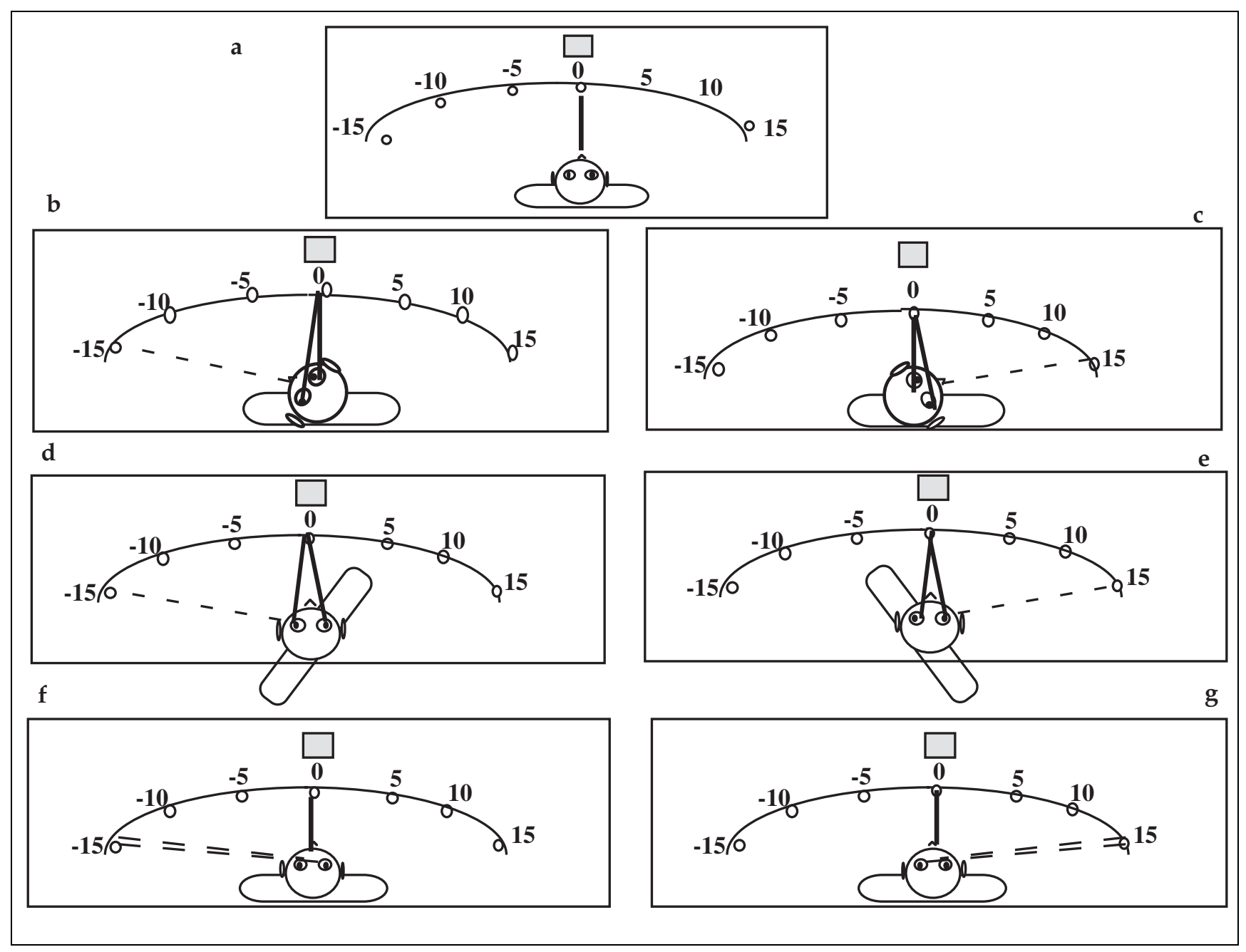

Figure 1. Schematic depiction of experiment for eye movement data collection with subject seated in the arc of LEDs and with speaker used to help elicit and maintain subjects' fixation: (a) baseline condition with midline of eyes, head, and trunk aligned with the environmental midline; (b) head left (HL) and (c) head right (HR) with the midline of the head rotated $15^{\circ}$ left or right but the midline of the eyes and trunk aligned with the environmental midline. The dashed line indicates the position of the head and the solid line the position of the eyes; (d) trunk left (TL) and (e) trunk right (TR) with the midline of the trunk rotated $15^{\circ}$ left or right but the midline of the eyes and the head aligned with the environmental midline. The dashed line indicates the position of the trunk and the solid line the position of the eyes and head; (f) eyes left (EL) and (g) eyes right (ER) with the midline of the eyes rotated $15^{\circ}$ left or right but the midline of the head and trunk aligned with the environmental midline. The dashed line indicates the position of the eyes and the solid line the position of the head and trunk.

acquired than those on the right, and there would be no difference between them. However, if eye movements are modulated by orbital position, then we might see the following, compared to the baseline: a speed-up in SRT for the targets at the same retinal position when the eyes are deviated ipsilesionally (Vuilleumier et al., 1999; Kooistra \& Heilman, 1989) (left panel) and a slowing of SRTs when the eyes are deviated contralesionally (right panel).

In each of the seven conditions shown in Figure 1, a block of trials was run with each target position randomly and equally sampled. On each trial, subjects maintained fixation (and this was ensured both by having the fixation point flash and a concurrent auditory signal emitted from a speaker located behind the fixation point). After a variable stimulus onset asynchrony (SOA) of 200,800 , or $1400 \mathrm{msec}$, imposed to ensure that subjects could not anticipate the target onset, a target appeared until a saccade was made. Both accuracy and SRT were measured.

The lesion sites of the patients are shown in Figure 3, and the autobiographical, neglect, and lesion details are included in Table 1. Patients 3, 4, and 5 have lesions directly implicating the parietal cortex, Patient 1 has some parietal damage although less extensive, and Patient 2 has extensive thalamic damage, essentially deafferenting the parietal cortex and precluding it from contributing to behavior. Additional methodological details are described in Methods.

\section{RESULTS}

Once the invalid data points were removed, the remaining valid trials were classified as correct or incorrect. 
Figure 2. Hypothetical illustration of (a) sole influence of retinocentric frame on saccadic reaction time with slower initiation of saccades to the left than right of fixation, independent of the other egocentric reference frames; (b) modulation of the retinocentric effect by eye-in-head signal with facilitation in reaction time when the eyes are deviated ipsilesionally and slowing when the eyes are deviated contralesionally.

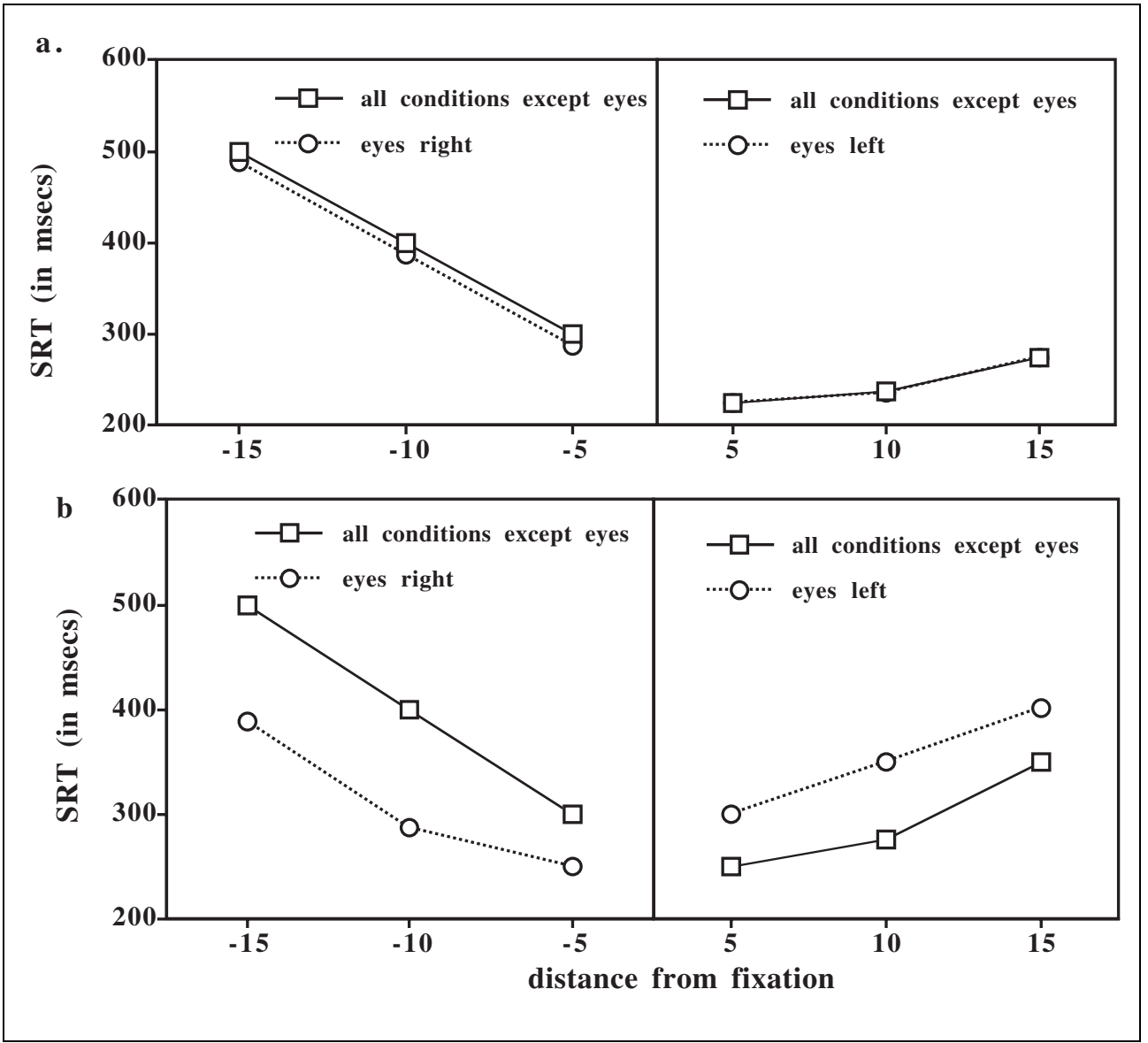

Separate analyses were performed on the errors and SRTs (correct trials only). We first report the group comparison (patients/controls) and then the data from the individual analyses. The analyses involve fitting a model and deriving the parameters that best characterize the data set. We adopted this procedure, rather than more standard analyses of variance, to characterize the entire dataset with the model parameters and evaluate the relative contribution of the different experimental conditions and target angles simultaneously. Note that each subject has their own slope and intercept: This allows us to take the individual data and variability into account, as well as the group average.

\section{Analysis of Error Data}

Two types of errors were identified: omissions, where the saccade did not occur within 1 sec after target onset, and direction errors, where a saccade was properly launched but in a direction opposite the target location. We consider the two types together, with the dependent measure being the number of errors as a proportion of the total trials (as subjects had differing number of trials). The proportion error was analyzed using a mixed effects logistic regression model, with two explanatory variables, condition, and target angle (distance from fixation). This latter measure is equivalent to the envi- ronmental angle for all conditions except the eye conditions (EL, ER) but to make comparisons across all conditions, we use distance from fixation as the standard measure. We assume that the errors for subject $i$, condition $k$, follows a binomial distribution with parameters $n_{i k}, p_{i k}$ where $n_{i k}$ is the total number of trials and $p_{i k}$ is the probability of making an error, and we model the error probability $p_{i k}$ as follows:

$$
\operatorname{logit}\left(p_{i k}\right)=\log \left(p_{i k} /\left(1-p_{i k}\right)\right)=\alpha+\beta_{i}+\gamma_{k}+\tau_{k} \mathrm{X}_{i k}^{\prime}
$$

where $p_{i k}$ is the probability of making an error of subject $i$, in condition $k, k=1, \ldots, 7 ; \alpha$ intercept; $\beta_{i}$ is the random intercept assumed to be $N\left(0, \sigma_{\beta}^{2}\right) ; \gamma_{k}$ is the main effect of experimental condition as deviation from baseline, $\gamma_{1}=0 ; \mathrm{X}^{\prime}$ is the target angle as distance from fixation; $\tau_{k}$ is the interaction of experimental condition and $\mathrm{X}^{\prime}$.

We use Bayesian methods to estimate the model parameters using BUGS (Spieghalter, Thomas, Best, \& Wilks, 1995). ${ }^{1}$ Note that the model is parameterized so that $\gamma_{k}$ are the deviations from the intercept of the B condition, while $\tau_{k}$ is the slope for condition $k$. The estimates of the coefficients ${ }^{2}$ for the two groups, as well as for each patient individually, are tabulated in Appendix A. For the controls, we report only those coefficients that differ from the patients rather than the full set of estimates as they made almost no errors. For individual 


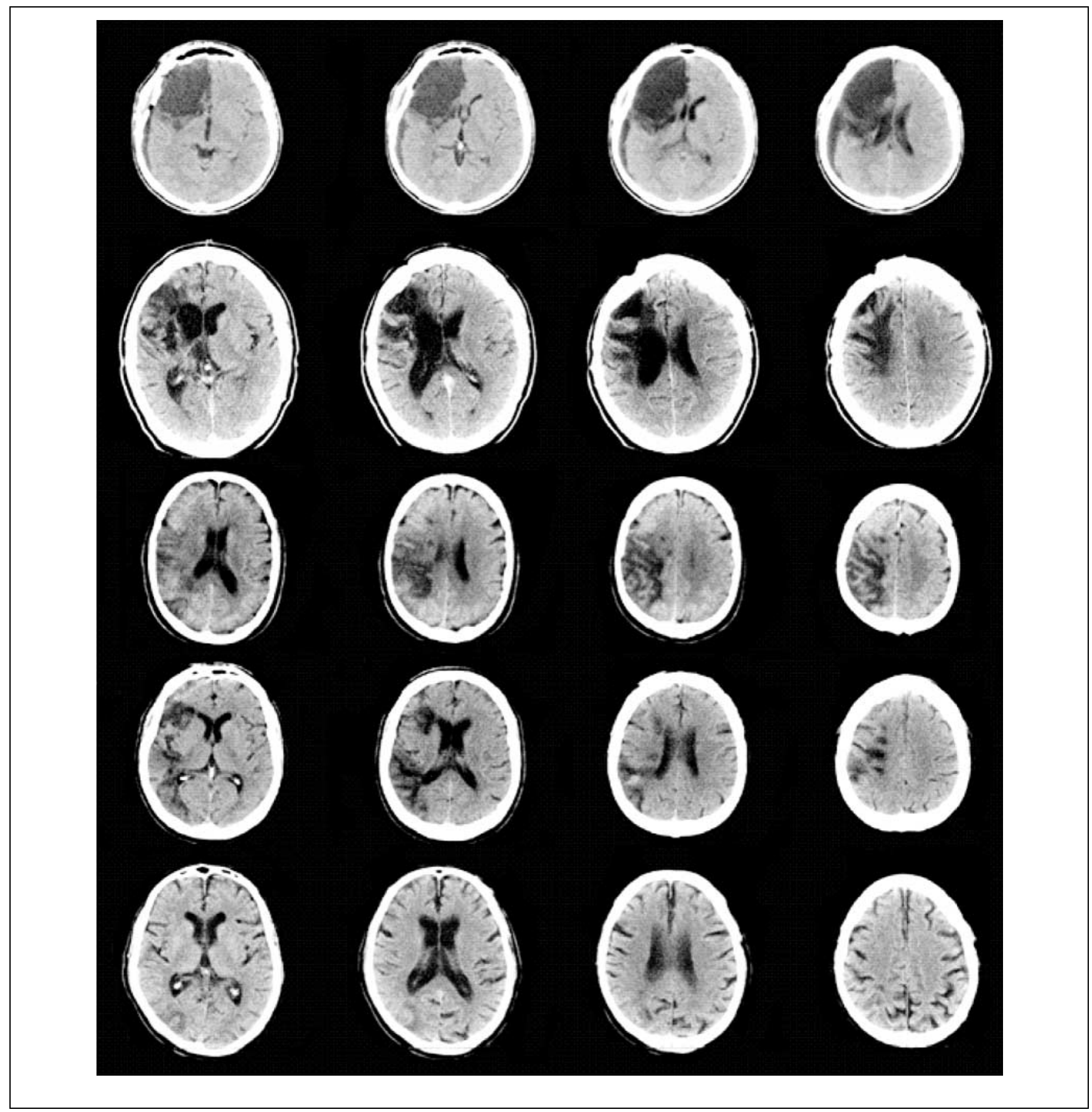

Figure 3. MRI scans for the five patients, depicting the location and extent of each subject's lesion. The last three patients have lesions directly implicating the parietal cortex. Left of image refers to right hemisphere.

data analysis, we used the same model as for the group but with no random effects.

The first important result is that, in the baseline (B) condition, there is an intercept difference for the patients, but not controls, for targets on the left versus right (see Figure 4). Additionally, there is a highly significant negative slope $\left(\tau_{1}=-0.127 \pm 0.013\right)$ for the patients, but not for the controls $\left(\tau_{1}=-0.014 \pm\right.$ 0.019), showing that, as the target is located further to the left, so the log-odds and probability of making an error increase.
We now consider only the patient data to evaluate the effect of the experimental manipulations on the error rates for left versus right targets. As is evident from Figure 5 (see Appendix A for the data), the intercepts and slopes in the head (HL, HR) and trunk conditions (TL, TR) do not differ significantly from the B condition, indicating that there is minimal, if any, influence of the head position and the trunk position on saccadic behavior.

The results from the eye rotation conditions and $\mathrm{B}$ are shown in Figure 6, for targets that share distance from fixation (ER: environment targets $10^{\circ}, 5^{\circ}$, and $0^{\circ}$ are 
Table 1. Patient Characteristics and Lesion Data for the Five Brain-Damaged Patients

\begin{tabular}{lllcllrr}
\hline Patients & Age & Lesion Site & Volume & $\% 39^{\mathrm{a}}$ & $\% 40^{\mathrm{a}}$ & Time Test $^{\mathrm{b}}$ & Neglect Score $^{\mathrm{c}}$ \\
\hline (1) RD & 22 & Frontoparietal & 252 & None & $<10$ & 7 & 107 \\
(2) JM & 52 & Thalamus & 126 & None & None & 48 & 111 \\
(3) JB & 66 & Parietal & 123 & $50-89$ & $>90$ & 17 & 103 \\
(4) JS & 67 & Parietal & 166 & $50-89$ & $50-89$ & 4 & 97 \\
$(5) \mathrm{RB}$ & 63 & Parietal & 114 & $10-49$ & $<10$ & 33 & 114 \\
\hline
\end{tabular}

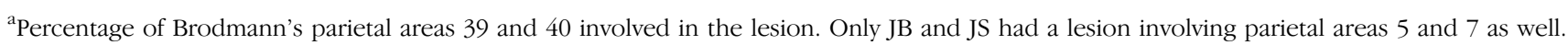

${ }^{\mathrm{b}}$ Time of testing, post onset in months.

${ }^{\mathrm{c}}$ Neglect score (maximum $=146$ ).

compared to $\mathrm{B}-5^{\circ},-10^{\circ}$, and $-15^{\circ}$; EL: environmental targets $-10^{\circ},-5^{\circ}$, and $0^{\circ}$ are compared to $\mathrm{B} 5^{\circ}, 10^{\circ}$, and $15^{\circ}$ ). In contrast to the head and trunk conditions and to $\mathrm{B}$, there is no significant slope in either the EL or ER condition. This indicates that, in the EL condition, when targets are to the right of gaze, the probability of making an error is low $(\gamma=-0.971 \pm 0.39)$, and this is equivalent across all target angles. Similarly, in the ER condition, when targets are to the left of gaze, the probability of making an error is high $(\gamma=1.777 \pm$ $0.345)$ but, again, roughly equal across all target angles.

In the individual analyses, Patients 2, 4, and 5 exhibit similar profiles and capture the critical aspects of the group (see Appendix A). They have significant negative slopes for B, as well as for the HL, HR, TL, and TR conditions; as the target is located further left, the logit increases. In addition, there is no significant slope in any of them in the EL or ER condition. The results for Patients 1 and 3 do not quite mirror the group average to the same extent. Their log-odds are fairly constant across conditions; they have significant intercepts in $\mathrm{B}$, but there is no slope aside from small increments for HL for Patient 1 and for TL for Patient 3. The absence of slopes for EL and ER in these last two patients is, however, consistent with the group data.

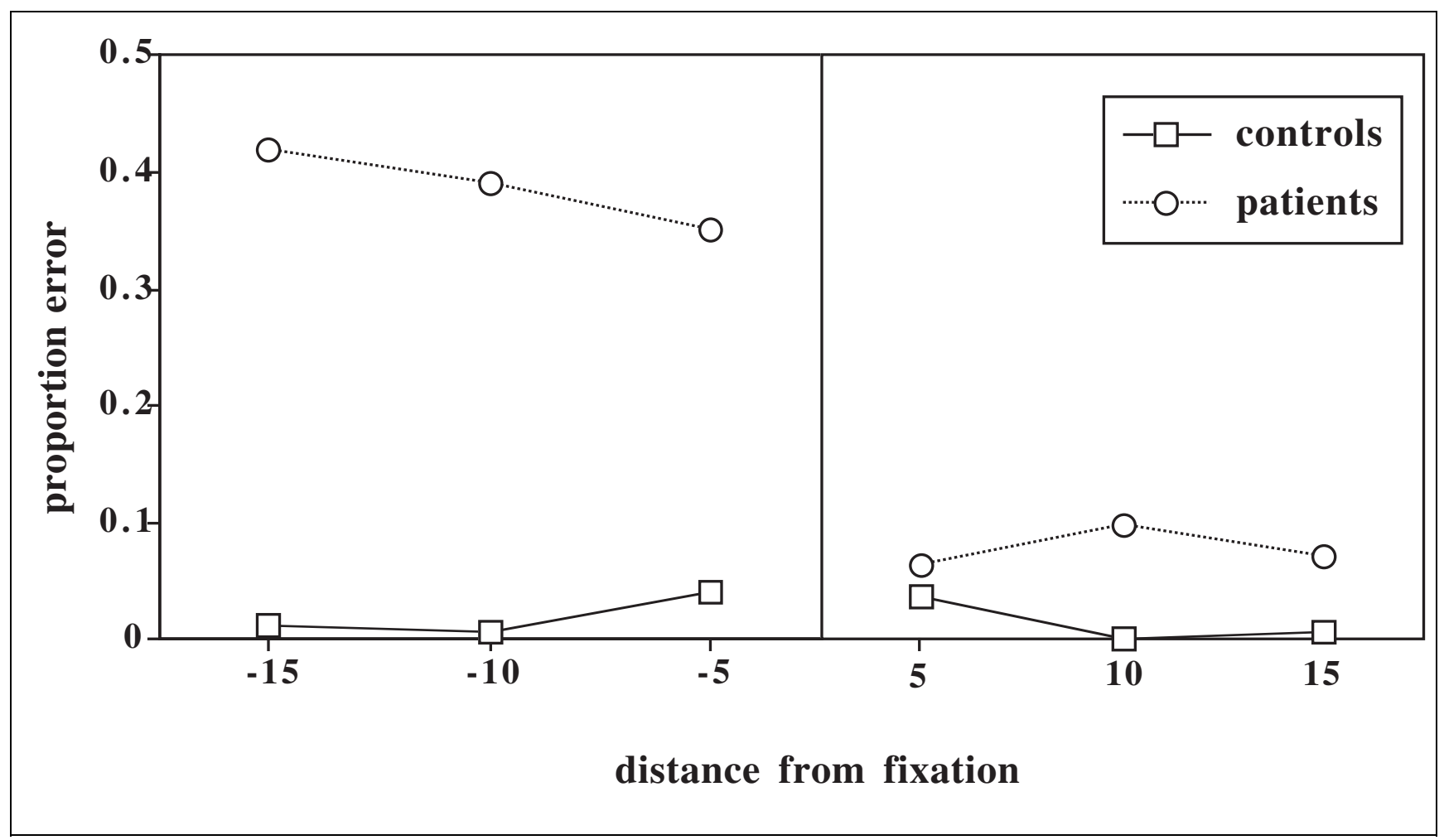

Figure 4. Mean proportion error for the patients and control subjects in the baseline condition for left and right targets as a function of distance from fixation. 


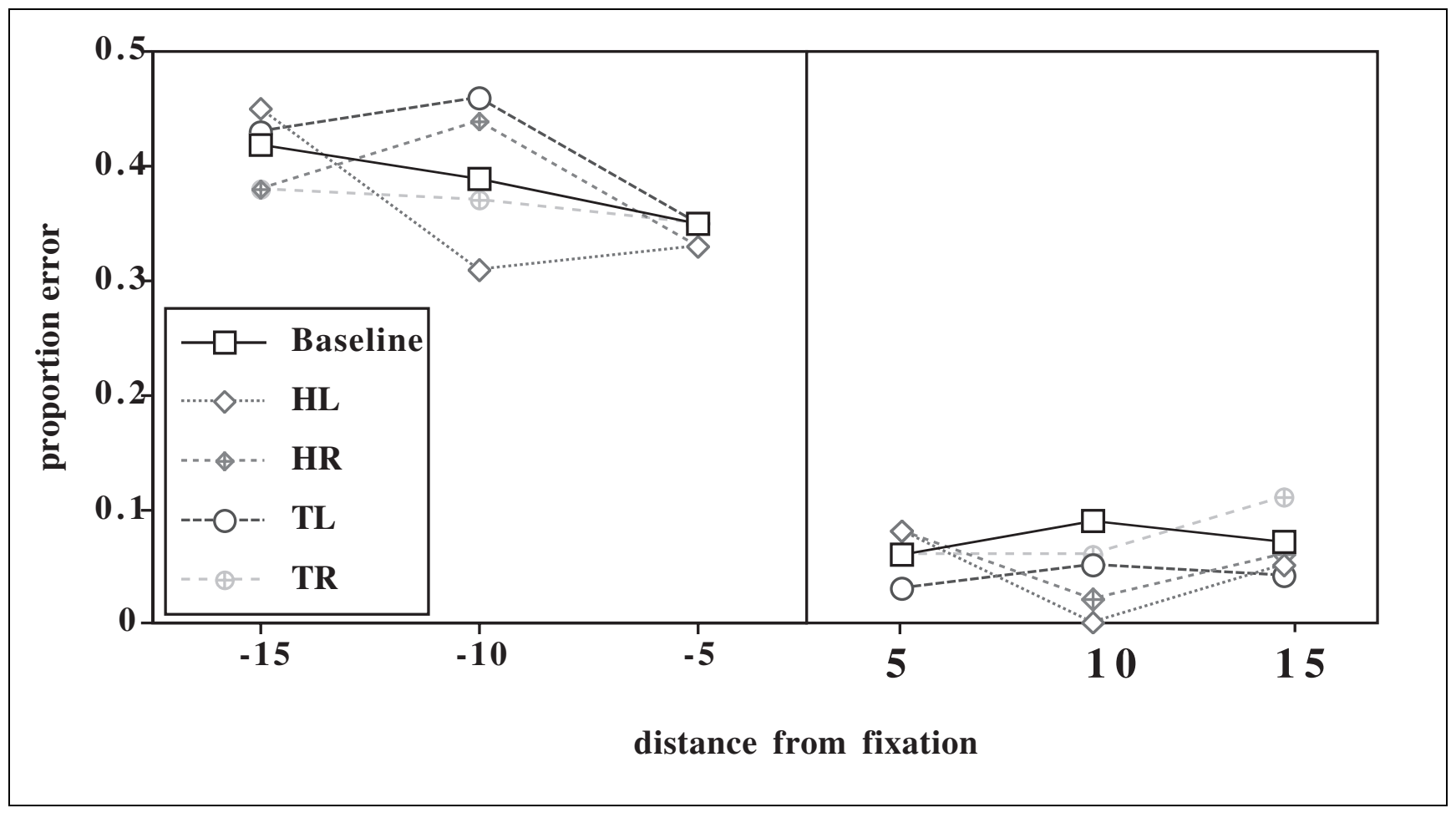

Figure 5. Mean proportion error for the patients in the baseline condition, as well as in the head and trunk manipulations, for left and right targets as a function of distance from fixation.

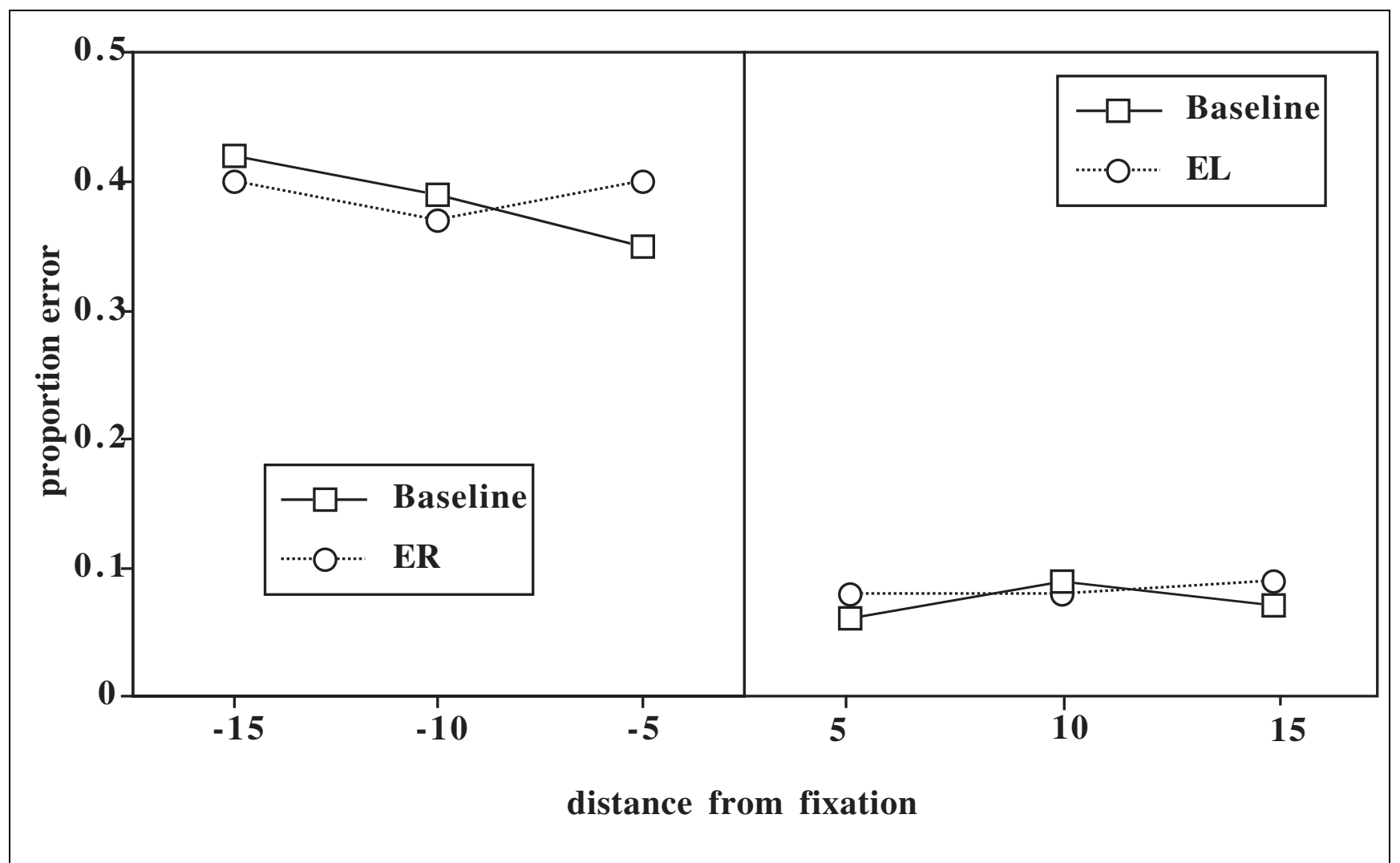

Figure 6. Mean proportion error for the patients in the baseline condition and eye rotation conditions for targets as a function of distance from fixation. 
Figure 7. Mean saccadic reaction time for the patients and control subjects in the baseline condition for left and right targets as a function of distance from fixation.

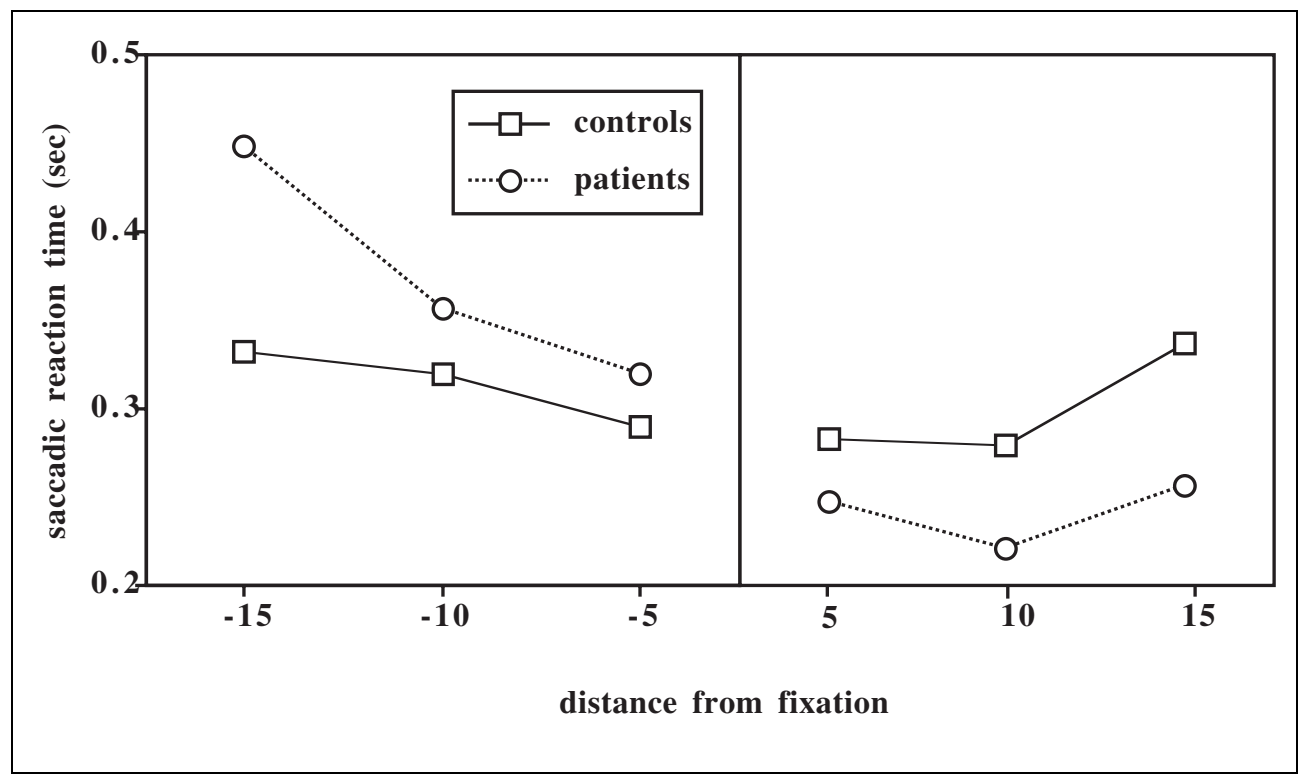

\section{Analysis of SRT}

We first transformed the SRT data (correct trials) to a log scale to adjust for unequal variance and the nonnormality of the error terms. As before, the two explanatory variables are target angle (distance from fixation) and condition. To examine the effects of conditions as a function of target angle, we fit the following normal linear mixed-effect model with repeated measures on the same subject as follows:

$$
Y_{i k j}=\alpha+\alpha_{i}+\gamma_{k}+\beta \mathrm{X}_{i k j}^{\prime}+\beta_{k} \mathrm{X}_{i k j}^{\prime}+\tau \mathrm{X}_{i k j}^{\prime}+\varepsilon_{i k j}
$$

where $\mathrm{Y}_{i k j}$ is the log reaction time for subject $i, i=1, \ldots$, 4 , condition $k, k=1, \ldots, 7$, replication $j, j=1, \ldots, n_{i k}$; $\mathrm{X}^{\prime}$ the retinotopic angle; $\alpha$ is the grand mean assumed to be the baseline (B) condition; $\alpha_{i}$ the random effects assumed to be $\sim N\left(0, \sigma_{\alpha}^{2}\right) ; \gamma_{k}$ the effects of the experimental condition, expressed as a deviation from the $\mathrm{B}$ condition, $\gamma_{1}=0 ; \beta$ the common slope; $\beta_{k}$ the interaction of $\mathrm{X}$ and experimental condition expressed as a deviation from the $\mathrm{B}$ condition, $\beta_{1}=0 ; \tau$ the quadratic effect of the retinotopic angle; and $\varepsilon_{i k j}$ the error term assumed to be $\sim N\left(0, \sigma_{\alpha}^{2}\right)$.

The model is parameterized so that the coefficient for each condition and interaction with retinotopic angle represent increases in intercept and slope from B (Appendix B). The individual analyses use the same model as the group but without the random effects. The model parameter estimates are obtained using "proc mixed" (SAS Institute, 1991) using Restricted Maximum Like-
Figure 8. Mean saccadic reaction time for patients in the baseline condition, as well as in the head and trunk manipulations, for left and right targets as a function of distance from fixation.

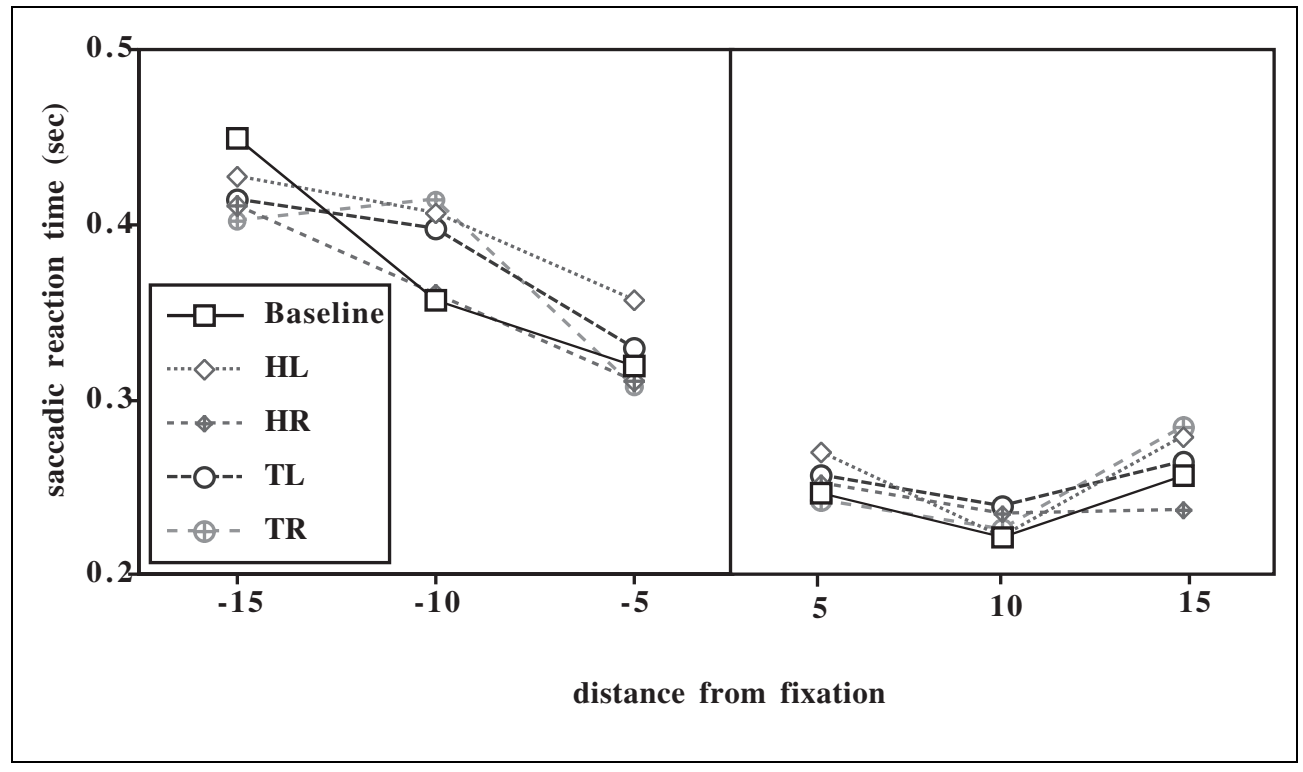


Figure 9. Mean saccadic reaction time for the patients in the baseline condition, as well as in the eye manipulations, for targets as a function of distance from fixation.

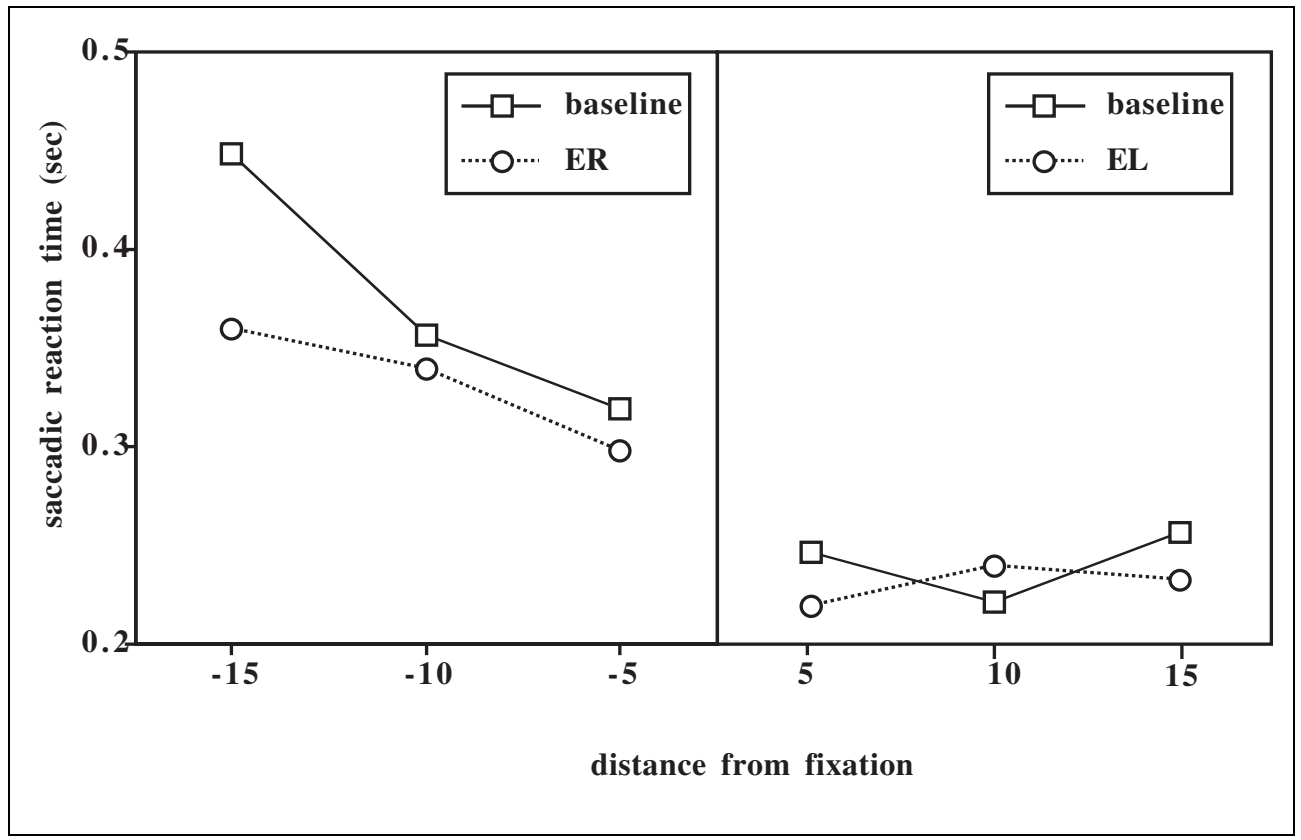

lihood method. We include a quadratic but not a cubic term as the latter does not contribute substantially to the fit of the model Bayesian Information Criterion (Pauler, 1998).

The first important result is that there is a highly significant negative slope for the patients in the $\mathrm{B}$ condition $(-0.0200 \pm 0.002)$, indicating that, as targets appear further to the left, SRT increases (see Figure 7). For the control subjects, the slope is not significantly different from zero $(-0.002 \pm 0.001)$, suggesting that the log SRT is symmetrical around fixation. It is also interesting to note that, whereas the increase in SRT for patients is greater than for controls on the left, the converse holds true on the right where the patients now have significantly shorter times than the control subjects. This right-sided superiority is consistent with reports that describe better performance for patients than controls for ipsilesional targets, as predicted by a theory of a spatial gradient in neglect with increasingly enhanced activation for more ipsilesional targets (Kinsbourne, 1993; Làdavas, Petronio, \& Umilta, 1990). We should also note that both groups have a significant quadratic term in the model but that it is much larger in the patients $(0.0008 \pm 0.0001)$ than in the controls $(0.0005 \pm 0.00005)$. This indicates that as the target distance increases to the left or right, SRT increases, consistent with U-shaped eccentricity effects, but it does so to a greater extent in the patients, presumably because of the increased neglect with the more eccentric, contralesional targets.

Figure 8 shows the comparison of the head and trunk conditions against $\mathrm{B}$ for the patient group. As was the case for the error data, neither the intercept nor slope in any of these conditions differ significantly from B, indicating no significant effect of the rotation of the head or trunk. Although the group as a whole does not show significant effects of the head or trunk, Patient 4 has a mild effect of the head in the intercept and slope. This is the only evidence in the group for an additional effect of head coordinates on performance.

Figure 9 shows the results from the eye rotation conditions plotted against $\mathrm{B}$ for the patients only. In contrast with the head and trunk rotations, we now observe a significant effect of the ER condition but not of the EL condition, on the SRT. Note, however, that the effect of eye position is one of modulation; the basic retinocentric effect of longer SRTs to left than right targets is not reversed but only qualified.

Table 2 shows the 95\% confidence intervals, using a Bonferroni correction, for the pairwise comparison of the mean log SRT for the ER and EL conditions against the $\mathrm{B}$ condition. We denote with $\mu(x, y)$ the

Table 2. 95\% CI for Pairwise Comparison of Baseline and EL and Baseline and ER Conditions

\begin{tabular}{lccc}
\hline & Estimate & $S D$ & $95 \%$ CI \\
\hline Baseline and ER & & & \\
$\mu(\mathrm{ER},-15)-\mu(\mathrm{B},-15)$ & -0.355 & 0.088 & $(-0.566,-0.14)$ \\
$\mu(\mathrm{ER},-10)-\mu(\mathrm{B},-10)$ & -0.265 & 0.098 & $(-0.500,-0.03)$ \\
$\mu(\mathrm{ER},-5)-\mu(\mathrm{B},-5)$ & -0.248 & 0.062 & $(-0.396,-0.10)$
\end{tabular}

Baseline and EL

\begin{tabular}{llll}
$\mu(\mathrm{EL}, 5)-\mu(\mathrm{B}, 5)$ & -0.018 & 0.065 & $(-0.174,0.14)$ \\
$\mu(\mathrm{EL}, 10)-\mu(\mathrm{B}, 10)$ & -0.113 & 0.062 & $(-0.261,0.04)$ \\
$\mu(\mathrm{EL}, 15)-\mu(\mathrm{B}, 15)$ & -0.044 & 0.060 & $(-0.188,0.10)$ \\
\hline
\end{tabular}


mean $\log$ SRT in experimental condition $x$ and distance from fixation with $y$. The $\log$ SRT is significantly shorter in the ER than B condition for all targets, revealing a 46-msec facilitation, relative to $\mathrm{B}$, when the eyes are deviated to the ipsilesional side. There is also greater facilitation with increasing eccentricity with a 100-msec speed-up for the $-15^{\circ}$ target (see Figure 9). When the eyes are deviated contralesionally, however, there is no difference between the B and EL conditions.

We see a very similar pattern of performance in Patients 1 through 3 (using the same linear model as the group): All of them have a highly significant negative slope in the $\mathrm{B}$ condition $(p<.001)$ and a highly significant positive increment in slope when the eyes are deviated to the right. With the exception of Patient 5 who only shows a trend, all patients show a highly significant quadratic term $(p<.001)$. Patients 4 and 5 appear to be different from the others at least as far as the negative baseline slope is concerned, but this may be artifactual. The model is parameterized so that the intercept and slope represent deviations from the $\mathrm{B}$ condition. If $\mathrm{B}$ is unstable for some reason, the coefficients will be markedly affected. Patient 4 has only a single observation in the $\mathrm{B}$ condition at $-15^{\circ}$, and Patient 5 makes many errors on the left at all angles. Because of the high error rate, their baselines are unstable. The slopes obtained from their data are positive but their overall behavior is consistent with that of the other three patients. This is confirmed by the fact that if we base the linear mixed-effect model on the data from the other patients only, the estimates of the fixed effects do not change compared with the case when all five patients are included.

\section{DISCUSSION}

This study was designed to examine the reference frame(s) within which spatial positions are coded in the human parietal cortex. We took, as our starting point, evidence from neurophysiological studies with nonhuman primates, indicating that the amplitude of the response of parietal neurons is defined with respect to the current midline of gaze and, further, that it is modulated by the position of the eye in the orbit (Pouget \& Sejnowski, 1997b; Andersen et al., 1985). To determine whether there is parallel evidence for retinocentric coding of spatial position and for modulation of this by an eye-in-head signal in humans, we compared the accuracy and SRT in five patients with left-sided neglect and control subjects to targets on the relative left or right, defined with respect to the midline of the eyes. To explore the contribution of other egocentric reference frames, we also examined whether performance was affected when saccades were made to targets located to the relative left or right of the midline of the head or of the trunk.
If SRTs are calibrated in the context of a retinalbased reference frame, performance should always be poorer for targets to the left than right of fixation and be unaffected by the position of the target relative to the other egocentric midlines. Additionally, if there is modulation of this coding by the eye-in-head signal, when the eyes are deviated ipsilesionally, we would expect better performance than when the eyes are focused straight ahead even though, in both cases, saccades are made to targets on the relative left of the current gaze position. Similarly, when the eyes are deviated contralesionally, we might expect poorer performance than when the eyes are straight ahead (see Figure 2 for hypothetical data demonstrating these predictions).

The results of the study were fairly straightforward. There was no left-right discrepancy in the normal subjects on either accuracy or SRT and SRT obeyed the expected U-shaped function reflecting increasing latency with target eccentricity. For the patients, performance was significantly worse for left than right targets when the eyes, head, and trunk were all aligned with the environmental straight ahead. There were clear intercept differences for left versus right targets in both accuracy and SRT and, additionally, there was a negative slope in the SRT data indicating poorer performance as targets were located further contralesionally. Of interest is the fact that, whereas patients were slower for left targets compared to the nonneurological controls, they were faster than the controls for right targets. These findings are consistent with views in which a spatial gradient, with greater ipsilesional than contralesional activation, underlies neglect (Pouget \& Driver, 2000; Kinsbourne, 1994; Làdavas et al., 1990).

Having established the contralesional deficit in the eye movement pattern in the baseline condition, we now examine the data from the midline manipulations that disambiguate the reference frames. For the patients, there was no obvious influence of the target position defined with respect to the midline of the trunk or of the head compared with the baseline position, on accuracy or SRT, suggesting that target location is not critically defined by these body postures. The major finding, however, was that SRT was significantly influenced by position defined with respect to the retinal midline such that targets to its left were always more poorly acquired than targets to its right. The second result was the effect of eye position; when the eyes were deviated to the right in the orbit, performance improved compared with the baseline even though, in both cases, targets fell to the left of fixation. The effect of gaze deviation was not evident when the eyes were deviated to the left and targets fell to the right of the line of gaze, presumably because SRTs to targets to the right of fixation were already at ceiling and there was no opportunity for further change. 
Taken together, these data provide the answers to the questions that we posed. There is clear evidence for spatial position coding that is defined oculocentrically. In addition, there is support for the claim that, as in nonhuman primates, there is an influence of the eye-inhead signal on behavior. These findings are compatible with previous neuropsychological studies, which find that unilateral neglect may be centered on the line of sight, as well as with those studies that show the influence of eye rotation on target detection (Vuilleumier et al., 1999; Nadeau \& Heilman, 1991; Kooistra \& Heilman, 1989). The findings also merge well with recent studies with normal subjects showing, for example, that the reference frame operating in an inhibition of return paradigm is oculocentric (Abrams \& Pratt, 2000) and that spatial priming is robust when the cue and target share the same retinal position (Barrett, Bradshaw, Rose, Everatt, \& Simpson, 2001). Additionally, a contribution of eye position with respect to the head has also been observed in a recent study with normal subjects (Karn, Möller, \& Hayhoe, 1997).

That we obtain oculocentric effects and modulation by eye position may not be that surprising given that the effector used in this study is the oculomotor system. As such, these findings reinforce the claim that spatial position may be coded with respect to more than one reference frame but that the effector system successfully exploits the spatial coding that is most appropriate for it (Colby, 1998; Snyder et al., 1997; Rizzolatti \& Camarda, 1987). It is important to bear in mind, then, that under other task conditions in which different outputs are required (e.g., limb movements), coordinates that are not retinal might become more influential. Although the heuristic, in which coordinates and effectors are matched, might hold in general, the situation is likely to be more complicated. For example, there are now data supporting retinocentric coding of auditory targets (Stricanne et al., 1996), a particularly interesting result given that we can localize auditory spatial positions with our eyes closed, and retinocentric coding and eye position modulation for limb-effector reaching tasks (Batista et al., 1999; see also DeSouza et al., 2000; Pouget et al., 2001).

Before considering the full implications of our results further, we need to consider two studies whose findings are apparently at odds with our data. In one study, Duhamel, Goldberg, Fitzgibbons, Sirigu, and Grafman (1992) describe a patient whose SRTs were $78 \mathrm{msec}$ slower to left than right targets but who showed no modulation by the orbital position of the eyes. Based on these data, they argued that the eye movement deficit arises solely with respect to retinocentric coordinates and that eye-in-head position is irrelevant. One possible reason for the discrepancy between their findings and ours is that their patient had an extensive frontal and parietal lesion; this may render a comparison between the studies illegitimate in the first place.
A second, perhaps more interesting reason is that Duhamel et al. only measured latencies for targets that appeared $5^{\circ}$ from fixation, whereas we sampled up to $15^{\circ}$. Because the modulation by the eye-in-head signal becomes more obvious at more eccentric locations where SRT is longest, it remains a possibility, that with additional sampling of more distant targets, modulation by orbital position might also have been observed in their patient.

Similar issues may arise in explaining the discrepancy between our findings and those of Karnath et al. (1991, 1993, 1996), although a further exploration of their data suggests that their findings might not be as discrepant as they appear to be on the surface. Using a similar method to ours, Karnath et al. found that the major influence on their patients' SRTs was the position of the target defined with respect to the midsaggital plane of the trunk, and that there was no effect of retinocentric coding nor a modulation by eye position (even though they used an eye movement task). In contrast, we observe no influence of the trunk midline on the group data nor on any of the individual analyses. As is always a problem, their patients differ from ours in lesion location; for example, some of their patients have lesions that include the frontal (Patient R2, 1991) or frontal with basal ganglia (MB, 1993) or frontal with parieto-occipital junction (AD, 1993) regions. Exactly what effect these neuroanatomical differences have is unclear, but the group differences are rather striking. A further possible difference across the studies concerns the experimental setting. Several of our subjects were uncomfortable sitting in total darkness with the result that we used a very small amount of floor lighting in the room. Although it is difficult to know how this may alter the presence of trunk midline influences, it may be the case that when subjects have some information about spatial position with respect to the environment, as in our situation, the reliance on other, perhaps less stable egocentric coordinates (which move with change of the observer) may diminish. Finally, whereas we sample multiple locations, they sampled a single location; as suggested above, the modulation by eye position becomes more apparent with greater target eccentricity.

Despite these differences, there remain important similarities across the Karnath et al. studies and the present data. First, a deeper exploration of the findings from their study suggests there may indeed be an influence of target position defined relative to the retinal axis, even though they argue that the trunk midline determines left and right and, hence, neglect. Note that in their data, there is no obvious effect of the trunk rotation for targets in the right visual field (Karnath et al., 1993). The absence of this effect suggests that targets to the right of the retinal midline are well detected, independent of trunk position and, as such, indicate a retinal axis effect. For targets in the left visual field, there is an effect of trunk position, but 
even here, it appears that the probability of detection was not entirely determined by the location of the target relative to the trunk. Rather, there was an interaction such that detection of the target in this field was better when the trunk was rotated to the left than in the baseline but not as accurate as detection of the right visual field target in the baseline condition. This suggests that targets to the left of the eyes were not as well acquired as those to the right of the eyes. This interaction suggests that it is not solely the midline of the trunk that determines what is left and right but that some additional spatial coordinates may be influencing target detection, and these coordinates may be oculocentric in nature. In sum, these data might be interpreted to indicate that targets are defined oculocentrically but can be modulated by the posture of the trunk (see Pouget \& Sejnowski, 1999 for a similar perspective on these data).

A second similarity between the current data and those of Karnath et al. concerns the absence of any clear modulation by head position (see also Vuillemier et al., 1999), although there is a clear representation of positions in a head-centered frame of reference in nonhuman primates. Duhamel, Bremmer, BenHamed, and Graf (1997), for example, have shown that neuronal activity in area VIP is not only modulated by eye-position but also by head-position signals (see also Brotchie, Andersen, Snyder, \& Goodman, 1995 for similar evidence). Thus, the neurons encode the azimuth and/or elevation of the stimulus independent of the eye position, thereby representing spatial positions explicitly with respect to a head-based reference frame. On the surface, the absence of this head effect in humans is surprising; when the eyes are deviated to the right (ER) and the head is aligned with zero, for example, the angular disparity between the eye and head midline is $+15^{\circ}$. This same angular disparity is found when the head is deviated to the left and the eyes remain straight ahead (HL). These two situations appear comparable and, yet, different results are obtained (compare Figures 8 and 9), suggesting that, even in our data, the eye midline and orbital position may not constitute a sufficient explanation of the observed pattern. Further consideration of the two situations, however, reveals that even if angular disparity is held constant, the situations are not truly comparable. When the head is deviated left, for example, as opposed to straight ahead, there is additional sensory input from the lengthening of the neck muscles. This proprioceptive information may assist subjects in elaborating an egocentric frame of reference taking head position into account (Biguer, Donaldson, Hein, \& Jeannerod, 1988). Alternatively, we might consider gaze angle as defined by eye position (eye-in-head signal) + head position (head-on-trunk signal), a definition that is formally accurate. In the head rotation conditions, then, the gaze angle remains $0^{\circ}$ because the head rotation and eye rotation cancel each other out. In the eye rotation conditions, however, the gaze angle is not equal to $0^{\circ}$ and coincides with the eye rotation. This difference might explain the absence of a head rotation effect and the presence of an effect for eye rotation. These results would also be consistent with a modulation by gaze angle rather than just by orbital position.

Before concluding, one final issue needs to be addressed. We have argued for oculocentric coding of spatial position and modulation by orbital position, based on the fact that left retinal targets are always slower than right targets, but SRTs in the ER condition are better than in the baseline. We do, however, need to consider an alternative explanation. The benefit in the ER condition might emerge not from the orbital signal per se, as we have argued, but rather from an absence of competition from targets in the right visual field in this condition. Note that, in the B condition, targets appear randomized to the left and right (of all reference frames) in the block of trials, but in the ER condition, targets always appear to the left of the retinal midline although they are to the left and right in the other reference frames. It is now well known that neglect can be ameliorated by reducing the competition between left and right targets, and this, rather than eye position signal, may explain the ER facilitation, relative to $B$. Note that, on every trial, irrespective of condition, there are always two stimuli present (the fixation LED and the target LED) and so there is always competition on a trial-by-trial basis. Thus, both in the ER and baseline conditions, a more rightward fixation LED is competing with the more leftward target and the conditions are equivalent in this regard. The differences come in across the block of trials, where competition is potentially reduced in the ER, relative to the baseline condition. Two factors argue against the interpretation of the data as arising from a difference in competition. One factor concerns the subjects' ability to exploit the contingencies: At the beginning of the experiment in the ER condition, subjects do not know that there will be only targets on the left of the eye midline; only with time will this contingency become apparent to them and only then will the competition be reduced. To examine whether the facilitation observed in the ER condition, relative to the baseline, only emerges with time or is present from the onset of the experiment (as predicted by the eye-in-head signal argument), we reanalyzed the entire data set, using time as a variable. To do so, we compared the SRTs for the ER and $\mathrm{B}$ conditions, including target angle and session as variables (Session 1 set against subsequent sessions). Most important is that the difference between the ER and baseline conditions is equivalent across early versus later sessions $[F(1,2)=3.9, p=$ $.18]$, and there is no interaction between these variables and target angle $[F(2,4)=.28, p>$.7]. This suggests that the facilitation in the ER condition is 
present early on, even before the contingency of target presentation is fully manifest. The second reason to reject this alternative explanation is that there is no difference between the baseline and EL condition; just as the ER benefit might have emerged from competition reduction, so one might have expected slower SRTS when competition is present (baseline) compared to when it is not (EL), but this is not so. Because the data do not support this alternative perspective, we adhere to the original claim and argue that, in humans, spatial position is coded with respect to the retinal midline and modulated by orbital position.

In conclusion, we have obtained evidence for the mediation of spatial representations by a set of coordinates aligned with the retinal axis and for the further modulation of this effect by the position of the eyes in the head. This intermediate representation of space, formed by combining information from various modalities, is one example of a host of increasingly abstract representations of space interposed between stimulus input and motor output (Pouget \& Snyder, 2000). Although the derivation of multiple intermediate spatial representations is an attractive solution to the computation of spatial position for various forms of action, there is one challenging aspect of this theory and that concerns the possible combinatorial explosion. If all possible inputs can be combined and then be accessible for all possible outputs, the system rapidly becomes computationally intractable. One solution to this problem has been to examine possible constraints so that all pairwise computations need not be computed. Thus, for example, there appears to be no direct evidence for a representation that combines vestibular and auditory input. Further empirical studies have suggested other constraints on the system. For example, it appears that a retinocentric representation plays a central role not only in the coding of visual space but also in the coding of information from the auditory and somatosensory modality. Thus, a retinocentric representation may serve as the foundation across different sensory modalities, allowing for easier communication across different sensory modalities, as well as across different output modalities. Consistent with neurophysiological data from nonhuman primates, our evidence suggests that the human parietal cortex utilizes intermediate representations and that one critical ingredient is a reference frame centered on the eye and modulated by an eye-in-head position signal.

\section{METHODS}

\section{Subjects}

All subjects were right-handed, had normal or correctedto-normal vision (see below), and consented to participate. Because the SRT can vary greatly with age, with older subjects exhibiting longer SRTs (Abrams, Pratt, \& Chasteen, 1998), we included a control group of nonneurological subjects against which to compare the patient data.

\section{Control Subjects}

Ten control subjects ( 3 men, 7 women), with a mean age and education of 71 and 15.6 years, respectively, were recruited through the Academy of Lifelong Learning program at Carnegie Mellon University, and none had a history of neurological disease nor hemispatial neglect, measured on a neglect battery (Black, Vu, Martin, \& Szalai, 1990). Three subjects were tested with glasses.

\section{Neurological Subjects}

Five men, one of whom was tested with glasses, participated. All exhibited hemispatial neglect, scoring below the 146 maximum cut-off for normal performance on the Behavioral Inattention test (Wilson, Cockburn, \& Halligan, 1987). No subject was hemianopic as revealed in visual field testing, described below.

\section{Experimental Apparatus}

The experiment was conducted in a windowless room, with the walls and ceiling painted optical flat black. The room was dark except for two dim nightlights on each side of the subject. The apparatus consisted of a table, a chinrest mounted on the table, and a chair on castors, all facing an array of LEDs, located along an arc of radius 1 $\mathrm{m}$ and centered at the chinrest/table midline. The electronic apparatus consisted of two parts. The first consisted of the LED array and an IBM-PC, which controlled their activation by reading from a file the sequence and duration of LED activation in each condition. The individual LEDs were illuminated via a computer-triggered signal. The second system, connected to electrodes placed around the subject's eyes, was an IBM-PC equipped with dedicated software for the acquisition of electrooculographic (EOG) data. EOG measures shifts in the electromagnetic dipole generated by voltage differences between the cornea and the retina. It is the most practical eye movement recording technique, does not require visualization or tracking of the eye per se, and is linear for movements up to $\pm 30^{\circ}$ (Young \& Sheena, 1975). Accuracy with surface electrodes is roughly $1-2^{\circ}$.

The data analysis was performed off-line, using a Microsoft Windows-based software program, which allows for trial-based calibration of the eye-movement recordings (i.e., the conversion from voltage data to eye position in terms of visual angle) and for the automatic computation of parameters such as SRT, amplitude, accuracy, velocity, duration, etc. Trial-by-trial calibration was chosen to reduce the effects of signal 
drift or of head/trunk movements, which might have occurred during the saccade recording.

\section{Procedure}

Subjects completed an eye exam (using a stereo optical industrial vision tester) to document their eyesight and to determine whether testing had to be performed with or without glasses, where relevant. Subjects with Snellen Equivalents of $20 / 100$ or better were tested without glasses. The seven EOG electrodes were then applied: one to the left and right of each eye (to monitor eye movements), one above and below the left eye (to monitor blinks), and one centered on the forehead (ground); the electrode impedances were tested to ensure proper electrical connection.

Subjects were then seated in the chair, which was rolled under the table until the subject's body contacted the edge of the table. A strap going around the table and secured behind the subject's chair minimized movements of the chair during testing. The subject's head was positioned in the chinrest, and a strap held it in place to minimize head movements. Once the subject was positioned, calibration of the equipment was performed. At this stage, we also verified that the subject could move their eyes to all locations and that the equipment was correctly recording the corresponding eye movements by having the subject saccade to LEDs that were activated in a random sequence.

The study measured saccades in a 1-D space defined by 7 locations organized along a circumference of 1-m radius (with the subject at its center) and spanning $30^{\circ}$ of visual angle. The 7 locations included a position at $0^{\circ}$ defined with respect to the screen, and three positions to the right and to the left, each $5^{\circ}$ apart. The positions are labeled $0^{\circ}, 5^{\circ}, 10^{\circ}, 15^{\circ},-5^{\circ},-10^{\circ},-15^{\circ}$. The positions were sampled in 7 different conditions (see Figure 1), which allowed a full comparison of the egocentric representations:

(a) Baseline condition (B): The eyes, head, and trunk midline were aligned with the screen $0^{\circ}$. Subjects fixated the $0^{\circ} \mathrm{LED}$, and targets at $5^{\circ}, 10^{\circ}$, and $15^{\circ}$ to the left and right were sampled.

(b) Head left (HL): The head was rotated so that its midline was aligned with the $-15^{\circ}$ location defined by the screen. The midlines of the trunk, eyes and screen were aligned. Subjects fixated the $0^{\circ}$ LED, and targets at $5^{\circ}, 10^{\circ}$, and $15^{\circ}$ to the left and right were sampled.

(c) Head right (HR): The head was rotated so that its midline was aligned with the $+15^{\circ}$ location defined by the screen. The midlines of the trunk, eyes and screen were aligned. Subjects fixated the $0^{\circ}$ LED, and targets at $5^{\circ}, 10^{\circ}$, and $15^{\circ}$ to the left and right were sampled. (d) Trunk left (TL): The trunk was rotated so that its midline was aligned with the $-15^{\circ}$ location defined by the screen. The midlines of the head, eyes, and screen were aligned. Subjects fixated the $0^{\circ} \mathrm{LED}$, and targets at $5^{\circ}, 10^{\circ}$, and $15^{\circ}$ to the left and right were sampled.

(e) Trunk right (TR): The trunk was rotated so that its midline was aligned with the $+15^{\circ}$ location defined by the screen. The midlines of the head, eyes, and screen were aligned. Subjects fixated the $0^{\circ}$ LED, and targets at $5^{\circ}, 10^{\circ}$, and $15^{\circ}$ to the left and right were sampled.

(f) Eyes left (EL): The eyes were rotated so that their midline was aligned with the $-15^{\circ}$ location defined by the screen. The midlines of the trunk, head, and screen were aligned. Note that fixation was at $-15^{\circ}$ and the targets sampled were $-10^{\circ},-5^{\circ}, 0^{\circ},+5^{\circ}$, $+10^{\circ},+15^{\circ}$ (environmental).

(g) Eyes right (ER): The eyes were rotated so that their midline was aligned with the $+15^{\circ}$ location defined by the screen. The midlines of the trunk, head, and screen were aligned. Note that fixation was at $+15^{\circ}$ and the targets sampled were $+10^{\circ},+5^{\circ}, 0^{\circ},-5^{\circ}$, $-10^{\circ},-15^{\circ}$ (environmental).

Note that the initial fixation point was always at $0^{\circ}$ defined by the screen, except where the midline of the eyes was decoupled. Under this condition, we sampled $0^{\circ}$ as a target, making the number of targets six (all locations in the environment except the current fixation point) as in the other conditions. The comparison is always between targets with the same retinal distance rather than environmental angle. Although one might compare, for example, $-5^{\circ}$, defined environmentally, in all conditions, when the eyes are deviated $15^{\circ}$ to the left, the target at $-5^{\circ}$ is now $10^{\circ}$ to the right of fixation. To make legitimate comparisons, then, we only compare performance on targets that share retinal angle.

The baseline condition was always tested first. To control for possible effects in the order of conditions, Latin square counterbalancing was used as far as possible for the three sets of decoupling conditions (eyes, head, and trunk), with random assignment of the left-right order in each set. Each block consisted of 54 trials with nine trials for each of the six targets, randomly sampled. Six controls completed two replications of the seven blocks, and the remaining four completed one replication. As much data was collected from each patient as possible as follows: Patient 1, three replications; Patient 2, five; Patient 3 did three throughout; and Patients 4 and 5 completed three replications of the baseline and two of the other six conditions.

Each trial had the following temporal sequence: The fixation light appeared, flashing intermittently and accompanied by an acoustic cue from the speaker behind 
Appendix A. Estimates $( \pm S D)$ of the Fixed Effects on the Error Data for the Group as a Whole and for Each Patient Individually

\begin{tabular}{|c|c|c|c|c|c|c|c|}
\hline & Description & Group & (1) $R D$ & (2) $J M$ & (3) $J B$ & (4) $J B$ & (5) $R B$ \\
\hline$\alpha$ & Grand mean & $-1.930(0.14)$ & -3.713 & -1.477 & -3.154 & -0.335 & 0.422 \\
\hline$\gamma_{2}$ & Inc. intercept EL & $-0.971(0.39)$ & -0.022 & 0.259 & 1.037 & -2.932 & -2.239 \\
\hline$\gamma_{3}$ & Inc. intercept ER & $1.777(0.36)$ & 1.590 & 1.971 & 2.003 & 2.384 & -0.690 \\
\hline$\gamma_{4}$ & Inc. intercept HL & $-0.214(0.22)$ & 1.063 & 0.133 & -0.299 & -0.057 & -0.761 \\
\hline$\gamma_{5}$ & Inc. intercept HR & $-0.056(0.21)$ & 0.616 & -0.102 & 1.338 & -0.045 & -0.649 \\
\hline$\gamma_{6}$ & Inc. intercept TL & $-0.078(0.19)$ & 0.641 & -0.340 & 0.713 & 0.064 & 0.021 \\
\hline$\gamma_{7}$ & Inc. intercept TR & $0.269(0.01)$ & 0.965 & 0.422 & 0.379 & -0.104 & -0.281 \\
\hline$\tau_{1}$ & Slope in B & $-0.127(0.02)$ & -0.003 & -0.097 & -0.060 & -0.295 & -0.091 \\
\hline$\tau_{2}$ & Slope in EL & $-0.001(0.02)$ & -0.003 & -0.014 & -0.020 & -0.006 & 0.025 \\
\hline$\tau_{3}$ & Slope in ER & $0.015(0.02)$ & 0.024 & 0.024 & 0.047 & 0.047 & -0.081 \\
\hline$\tau_{4}$ & Slope in HL & $-0.160(0.02)$ & -0.079 & -0.107 & -0.006 & -0.254 & -0.141 \\
\hline$\tau_{5}$ & Slope in HR & $-0.140(0.02)$ & -0.063 & -0.067 & -0.046 & -0.271 & -0.150 \\
\hline$\tau_{6}$ & Slope in TL & $-0.174(0.02)$ & -0.127 & -0.135 & -0.069 & -0.348 & -0.143 \\
\hline$\tau_{7}$ & Slope in TR & $-0.116(0.01)$ & -0.132 & -0.037 & -0.072 & -0.166 & -0.129 \\
\hline$\sigma_{\beta}^{2}$ & $\begin{array}{l}\text { Between-subjects } \\
\text { variability }\end{array}$ & $2.807(3.25)$ & & & & & \\
\hline
\end{tabular}

Inc. $=$ increase.

Appendix B. Estimates $( \pm S D)$ for Experimental Conditions Derived from Model of SRT Data

\begin{tabular}{|c|c|c|c|c|c|c|c|}
\hline & Description & Group & (1) $R D$ & (2) $J M$ & (3) $\mathrm{JB}$ & (4) $J S$ & (5) $R B$ \\
\hline$\alpha$ & Grand mean & $-1.3001(0.15)$ & -1.685 & -1.3366 & -0.9781 & -1.5396 & -1.0301 \\
\hline$\gamma_{2}$ & Inc. intercept EL & $-0.0137(0.03)$ & -0.1162 & -0.1197 & 0.1938 & 0.3960 & -0.1877 \\
\hline$\gamma_{3}$ & Inc. intercept ER & $0.0775(0.04)$ & 0.1535 & -0.1338 & 0.0607 & 0.8495 & -0.8978 \\
\hline$\gamma_{4}$ & Inc. intercept HL & $0.0543(0.03)$ & 0.1545 & -0.296 & 0.0281 & 0.4490 & -0.0190 \\
\hline$\gamma_{5}$ & Inc. intercept HR & $-0.0188(0.03)$ & -0.0572 & 0.00003 & -0.0736 & 0.4982 & -0.2177 \\
\hline$\gamma_{6}$ & Inc. intercept TL & $0.0291(0.03)$ & 0.01070 & 0.0325 & -0.0447 & 0.0721 & -0.1591 \\
\hline$\gamma_{7}$ & Inc. intercept $\mathrm{TR}$ & $0.0120(0.03)$ & 0.1003 & 0.0396 & -0.0343 & 0.0538 & 0.2264 \\
\hline$\beta$ & Slope (baseline) & $-0.0200(0.002)$ & -0.0223 & -0.0201 & -0.218 & 0.0148 & 0.0321 \\
\hline$\beta_{2}$ & Inc. slope EL & $-0.0061(0.003)$ & -0.0025 & 0.0048 & -0.0070 & -0.0528 & -0.0333 \\
\hline$\beta_{3}$ & Inc. slope ER & $0.0350(0.004)$ & 0.0442 & 0.0296 & 0.0293 & -0.0031 & -0.1350 \\
\hline$\beta_{4}$ & Inc. slope HL & $0.00001(0.003)$ & 0.0020 & -0.0039 & 0.0075 & -0.0344 & -0.0207 \\
\hline$\beta_{5}$ & Inc. slope HR & $0.0007(0.003)$ & -0.0028 & 0.0037 & 0.0006 & -0.0378 & -0.0169 \\
\hline$\beta_{6}$ & Inc. slope TL & $0.0015(0.003)$ & 0.0044 & -0.0007 & 0.0045 & -0.0064 & 0.0125 \\
\hline$\beta_{7}$ & Inc. slope TR & $0.0030(0.003)$ & 0.0017 & 0.0062 & 0.0074 & -0.0190 & -0.0159 \\
\hline$\tau$ & Quadratic effect & $0.0008(0.001)$ & 0.0008 & 0.0006 & 0.0007 & 0.0009 & 0.0004 \\
\hline$\sigma_{\alpha}^{2}$ & $\begin{array}{l}\text { Between-subjects } \\
\text { variability }\end{array}$ & $0.0874(0.07)$ & & & & & \\
\hline$\sigma_{\varepsilon}^{2}$ & $\begin{array}{l}\text { Within-subjects } \\
\text { variability }\end{array}$ & $0.1538(0.004)$ & & & & & \\
\hline
\end{tabular}


the fixation LED; after an 800-msec interval, the fixation light stopped flashing and the acoustic cue stopped; after a variable time interval (200, 800, or $1400 \mathrm{msec}$ SOA equally but randomly sampled), the target LED appeared; after a 1200-msec time interval, both lights (fixation and target) were turned off; after a 2000-msec intertrial interval, the fixation light appeared again to start another trial.

\section{Treatment of the Data}

The raw data were transferred to another computer equipped with the eye-movement data analysis software. Each trial was manually edited to review the results of the automated analysis of the saccadic parameters. Although the eye movements of both eyes were recorded, only one eye was considered: Before beginning the analysis, both eye recordings were examined and the one with lower noise levels or the one the subject indicated as the better eye was chosen. Saccades were identified using a velocity threshold algorithm that reliably detects saccades of $1^{\circ}$. Trials were considered valid when fixation was maintained for at least $100 \mathrm{msec}$ prior to the onset of the target and when the saccade occurred at least $70 \mathrm{msec}$ after target onset (to eliminate anticipatory saccades). Trials where fixation was not maintained, where the saccade occurred too early, where there were many blinks, or where calibration was not possible, were considered invalid and removed. In some trials, the target was reached with a multistep saccade, usually resulting from an initial hypometric saccade (Behrmann, Ghiselli-Crippa, \& Di Matteo, 2002; Heide \& Kömpf, 1998), and these were also considered invalid.

\section{Acknowledgments}

This work was funded by awards from the National Institute of Health (MH5424-06; CA 54852-08). The authors thank Jim Nelson and Thomas McKeeff for the help with data collection, Sarah Shomstein for the assistance with data analysis, Drs. Sandra Black and Peter Gao for digitizing the lesions and conducting the volumetric analysis of the patient scans and Drs. Carol Colby and Carl Olson for their valuable input. We also thank the patients and the participants from the Academy of Lifelong Learning at Carnegie Mellon University.

Reprint requests should be sent to Marlene Behrmann, Department of Psychology, Carnegie Mellon University, Pittsburgh, PA 15213-3890, USA, or via e-mail: behrmann+@cmu.edu.

\section{Notes}

1. We also fit this model using the macro "glimmix" in SAS, and the results were consistent with those of BUGS with the exception that larger standard errors were obtained with SAS than with BUGS.

2. The Bayesian estimates are the means of the posterior distribution of the parameters. The posterior distributions are simulated via Gibbs sampling, assuming the following (diffuse) priors for the model parameters: $\alpha \sim N(0,10) ; \gamma_{k} \sim N(0,10)$

$k=2, \ldots, 7 ; \tau_{k} \sim N\left(\xi, \sigma_{\tau}{ }^{2}\right) ; \xi \sim N(0,10) ; 1 / \sigma_{\beta}{ }^{2} \sim \Gamma(1.44,0.45) ;$ $1 / \sigma_{\tau}^{2} \sim \Gamma(1.44,0.45)$.

\section{REFERENCES}

Abrams, R. A., \& Pratt, J. (2000). Oculocentric coding of inhibited eye movements to recently attended locations. Journal of Experimental Psychology: Human Perception and Performance, 26, 776-788.

Abrams, R. A., Pratt, J., \& Chasteen, A. L. (1998). Aging and movement: Variability of force pulses for saccadic eye movements. Psychology and Aging, 13, 387-395.

Andersen, R. A. (1995). Encoding of intention and spatial location in the posterior parietal cortex. Cerebral Cortex, 5, 457-469.

Andersen, R. A., Essick, G. K., \& Siegel, R. M. (1985). Encoding of spatial location by posterior parietal neurons. Science, 230, 456-458.

Andersen, R. A., Snyder, L. H., Bradley, D. C., \& Xing, J. (1997). Multimodal representation of space in the posterior parietal cortex and its use in planning movements. Annual Review of Neuroscience, 20, 303-330.

Andersen, R. A., Snyder, L. H., Li, C.-S., \& Stricanne, B. (1993). Coordinate transformations in the representation of spatial information. Current Opinion in Neurobiology, 3, 171-176.

Barrett, D. J. K., Bradshaw, M. F., Rose, D., Everatt, J., \& Simpson, P. J. (2001). Reflexive shifts of covert shifts of covert attention operate in an egocentric coordinate frame. Perception, 30, 1083-1091.

Bartolomeo, P., \& Chokron, S. (2001). Levels of impairment in unilateral neglect. In F. Boller, \& J. Grafman (Eds.), Handbook of neuropsychology, (vol. 4, pp. 67-98). Amsterdam: Elsevier.

Barton, J. J. S., Behrmann, M., \& Black, S. E. (1998). Ocular search during line bisection: The effects of hemineglect and hemianopia. Brain, 121, 1117-1131.

Batista, A., Buneo, C., Snyder, L. H., \& Andersen, R. A. (1999). Reach plans in eye-centered coordinates. Science, 285, 257-260.

Behrmann, M. (2000). Spatial reference frames and hemispatial neglect. In M. Gazzaniga (Ed.), The cognitive neurosciences (2nd ed., pp. 651-666). Cambridge: MIT Press.

Behrmann, M., Barton, J. J. S., Watt, S., \& Black, S. E. (1997). Impaired visual search in patients with unilateral neglect: An oculographic analysis. Neuropsychologia, 35, 1445-1458.

Behrmann, M., Ghiselli-Crippa, T., \& Di Matteo, I. (2002). Impaired initiation but not execution of eye movements in hemispatial neglect. Behavioral Neurology, 13, 1-16.

Behrmann, M., \& Moscovitch, M. (1994). Object-centered neglect in patients with unilateral neglect: Effects of leftright coordinates of objects. Journal of Cognitive Neuroscience, 6, 1-16.

Behrmann, M., \& Tipper, S. P. (1999). Attention accesses multiple reference frames: Evidence from neglect. Journal of Experimental Psychology: Human Perception and Performance, 25, 83-101.

Beschin, N., Cubelli, R., Della Sala, S., \& Spinazzola, L. (1997). Left of what? The role of egocentric coordinates in neglect. Journal of Neurology, Neurosurgery and Psychiatry, 63, 483-489.

Biguer, B., Donaldson, I. M. L., Hein, A., \& Jeannerod, M. (1988). Neck muscle vibration modifies the representation of visual motion and direction in man. Brain, 111, 1405-1424.

Bisiach, E., \& Vallar, G. (2000). Unilateral neglect in humans. In F. Boller, \& J. Grafman (Eds.), Handbook of neuropsychology (2nd ed., vol. 1, pp. 459-502). Amsterdam: Elsevier. 
Black, S. E., Vu, B., Martin, D., \& Szalai, J. P. (1990). Evaluation of a bedside battery for hemispatial neglect in acute stroke. Journal of Clinical and Experimental Neuropsychology, 12, 102 [abstract].

Braun, D., Weber, H., Mergner, T., \& Schulte-Monting, J. (1992). Saccadic reaction times in patients with frontal and parietal lesions. Brain, 115, 1359-1386.

Brotchie, P. R., Andersen, R. A., Snyder, L. H., \& Goodman, S. J. (1995). Head position signals used by parietal neurons to encode locations of visual stimuli. Nature, 375, 232-235.

Calvanio, R., Petrone, P. N., \& Levine, D. (1987). Left visual spatial neglect is both environment-centered and bodycentered. Neurology, 37, 1179-1183.

Cate, A., \& Behrmann, M. (submitted). Hemispatial neglect: Spatial and temporal influences.

Chedru, F., Leblanc, M., \& Lhermitte, F. (1973). Visual searching in normal and brain-damaged subjects: Contribution to the study of unilateral subjects. Cortex, 9, 94-111.

Colby, C. (1998). Action-oriented spatial reference frames in cortex. Neuron, 20, 15-24.

Colby, C. L., Duhamel, J. R., \& Goldberg, M. E. (1995). Oculocentric representation in parietal cortex. Cerebral Cortex, 5, 470-481.

Colby, C. L., \& Goldberg, M. E. (1999). Space and attention in parietal cortex. Annual Review of Neuroscience, 22, 319-349.

DeSouza, J. F. X., Dukelow, S. P., Gati, J. S., Menon, R. S., Andersen, R. A., \& Vilis, T. (2000). Eye position signal modulates a human parietal pointing region during memory-guided movements. Journal of Neuroscience, 20, 5835-5840.

Duhamel, J.-R., Bremmer, F., BenHamed, S., \& Graf, W. (1997). Spatial invariance of visual receptive fields in parietal cortex neurons. Nature, 389, 845-848.

Duhamel, J. R., Colby, C. L., \& Goldberg, M. E. (1992). The updating of representations of visual space in parietal cortex by intended eye movements. Science, 225, 90-92.

Duhamel, J. R., Goldberg, M. E., Fitzgibbons, E. J., Sirigu, A., \& Grafman, J. (1992). Saccadic dysmetria in a patient with a right frontoparietal lesion: The importance of corollary discharge for accurate spatial behavior. Brain, 115, 1387-1402.

Farah, M. J., Brunn, J. L., Wong, A. B., Wallace, M., \& Carpenter, P. (1990). Frames of reference for the allocation of spatial attention: Evidence from the neglect syndrome. Neuropsychologia, 28, 335-347.

Gainotti, G. (1993). The role of spontaneous eye movements in orienting attention and in unilateral neglect. In I. Robertson, \& J. C. Marshall (Eds.), Hemispatial neglect (pp. 107-122). London: Erlbaum.

Girotti, F., Casazza, M., Musicco, M., \& Avanzini, G. (1983). Oculomotor disorders in cortical lesions in man: The role of unilateral neglect. Neuropsychologia, 21, 543-553.

Heide, W., \& Kömpf, D. (1998). Combined deficits of saccades and visuo-spatial exploration after cortical lesions. Experimental Brain Research, 123, 164-171.

Hillis, A. E., \& Rapp, B. (1998). Unilateral spatial neglect in dissociable frames of reference: A comment on Farah et al. Neuropsychologia, 36, 1257-1262.

Hornak, J. (1992). Ocular exploration in the dark by patients with visual neglect. Neuropsychologia, 30, 547-552.

Johnston, C. (1988). Eye movements in visual hemi-neglect. In C. W. Johnston, \& F. J. Pirozzolo (Eds.), Neuropsychology of eye movements (pp. 235-263). Hillsdale, NJ: Erlbaum.

Karn, K. S., Möller, P., \& Hayhoe, M. (1997). Reference frames in saccadic targetting. Experimental Brain Research, 115, 267-282.

Karnath, H. O., Christ, K., \& Hartje, W. (1993). Decrease of contralateral neglect by neck muscle vibration and spatial orientation of the trunk midline. Brain, 116, 383-396.
Karnath, H.-O., \& Ferber, S. (1999). Is space representation disorted in neglect? Neuropsychologia, 37, 7-15.

Karnath, H. O., \& Fetter, M. (1995). Ocular space exploration in the dark and its relation to subjective and objective body orientation in neglect patients with parietal lesions. Neuropsychologia, 33, 371-377.

Karnath, H. O., Fetter, M., \& Dichgans, J. (1996). Ocular exploration of space as a function of neck proprioceptive and vestibular input-observations in normal subjects and patients with spatial neglect after parietal lesions. Experimental Brain Research, 109, 333-342.

Karnath, H. O., \& Huber, W. (1992). Abnormal eye movement behaviour during text reading in neglect syndrome: A case study. Neuropsychologia, 30, 593-598.

Karnath, H. O., Schenkel, P., \& Fisher, B. (1991). Trunk orientation as the determining factor of the contralateral deficit in the neglect syndrome and as the physical anchor of the internal representation of body orientation in space. Brain, 114, 1997-2014.

Kinsbourne, M. (1993). Orientational bias model of unilateral neglect: Evidence from attentional gradients within hemispace. In I. H. Robertson, \& J. C. Marshall (Eds.), Unilateral neglect: Clinical and experimental studies (pp. 63-86). Hove, UK: Erlbaum.

Kinsbourne, M. (1994). Mechanisms of neglect: Implications for rehabilitation. Neuropsychological Rehabilitation, 4 , 151-153.

Kooistra, C. A., \& Heilman, K. M. (1989). Hemispatial visual inattention masquerading as hemianopia. Neurology, 39, $1125-1127$.

Làdavas, E. (1987). Is hemispatial deficit produced by right parietal damage associated with retinal or gravitational coordinates? Brain, 110, 167-180.

Làdavas, E., Pesce, M. D., \& Provinciali, L. (1989). Unilateral attention deficits and hemispheric asymmetries in the control of visual attention. Neuropsychologia, 27, 353-366.

Làdavas, E., Petronio, A., \& Umilta, C. (1990). The deployment of visual attention in the intact field of hemineglect patients. Cortex, 26, 307-317.

Mazzoni, P., Andersen, R. A., \& Jordan, M. I. (1991). A more biologically plausible learning rule for neural networks. Proceedings of the National Academy of Sciences, U.S.A., 88, 4433-4437.

Meienberg, O., Zangemeister, W. H., Rosenberg, M., Hoyt, W., \& Stark, L. (1981). Saccadic eye movement strategies in patients with homonymous hemianopia. Annals of Neurology, 9, 537-544.

Moscovitch, M., \& Behrmann, M. (1994). Coding of spatial information in the somatosensory system: Evidence from patients with right parietal lesions. Journal of Cognitive Neuroscience, 6, 151-155.

Nadeau, S. E., \& Heilman, K. M. (1991). Gaze dependent hemianopia without hemispatial neglect. Neurology, 41, 1244-1250.

Niemeier, M., \& Karnath, H.-O. (2000). Exploratory saccades show no direction-specific deficit in neglect. Neurology, 54, 515-518.

Pauler, D. K. (1998). The Schwartz criterion and related methods for normal linear models. Biometrika, 85, 13-217.

Pouget, A., \& Driver, J. (2000). Relating unilateral neglect to the neural coding of space. Current Opinion in Neurobiology, 10, 242-249.

Pouget, A., Ducom, J.-C., Torri, J., \& Bavelier, D. (2001). Multisensory spatial representations in eye-centered coordinates. (submitted).

Pouget, A., \& Sejnowski, T. (1999). A new view of hemineglect based on the response properties of parietal neurones. In N. Burgess, K. J. Jeffery, \& J. O'Keefe (Eds.), Spatial functions 
of the hippocampal formation and parietal cortex (pp. 127-146). Oxford, UK: Oxford University Press.

Pouget, A., \& Sejnowski, T. J. (1997a). Lesion in a basis function model of parietal cortex: Comparison with hemineglect. In P. Thier, \& H.-O. Karnath (Eds.), Parietal lobe contributions to orientation in 3D space (pp. 521-538). Heidelberg, Germany: Springer.

Pouget, A., \& Sejnowski, T. J. (1997b). A new view of hemineglect based on the response properties of parietal neurones. Philosophical Transaction of the Royal Society, 352, 1449-1459.

Pouget, A., \& Sejnowski, T. J. (1997c). Spatial transformations in the parietal cortex using basis functions. Journal of Cognitive Neuroscience, 9, 222-237.

Pouget, A., \& Sejnowski, T. J. (2001). Simulating a lesion in a basis function model of spatial representation: comparison with hemispatial neglect. Psychological Review, 108, 653-673.

Pouget, A., \& Snyder, L. H. (2000). Computational approaches to sensorimotor transformations. Nature Neuroscience, 3, 1192-1198.

Rapscak, S. Z., Watson, R. T., \& Heilman, K. M. (1987). Hemispace-visual field interactions in visual extinction. Journal of Neurology, Neurosurgery and Psychiatry, 50, 1117-1124.

Rizzolatti, G., Berti, A., \& Gallese, V. (2000). Spatial neglect: Neurophysiological bases, cortical circuits and theories. In F. Boller, \& J. Grafman (Eds.), Handbook of neuropsychology. Amsterdam: Elsevier.

Rizzolatti, G., \& Camarda, R. (1987). Neural circuits for spatial attention and unilateral neglect. In M. Jeannerod (Ed.), Neurophysiological and neuropsychological aspects of spatial neglect (pp. 289-313). North Holland: Elsevier.

SAS Institute (1991). Getting started with PROC MIXED. Cary, NC: SAS Institute.

Schindler, I., \& Kerkhoff, G. (1997). Head and trunk orientation modulate visual neglect. NeuroReport, 8 , 2681-2685.

Snyder, L. H., Batista, A. P., \& Andersen, R. A. (1997). Coding of intention in the posterior parietal cortex. Nature, 386, 167-170.
Snyder, L. H., Grieve, K. L., Brotchie, P., \& Andersen, R. A. (1998). Separate body- and world-referenced representations of visual space in parietal cortex. Nature, 394, 887-891.

Spieghalter, D. J., Thomas, A., Best, N. G., \& Wilks, W. R. (1995). BUGS: Bayesian using Gibbs sampling, version 5.0. Cambridge, UK: MRC Biostatistics Unit.

Stein, J. F. (1992). The representation of egocentric space in the posterior parietal cortex. Behavioral and Brain Sciences, 15, 691-700.

Stricanne, B., Andersen, R. A., \& Mazzoni, P. (1996). Eye-centered, head-centered and intermediate coding of remembered sound locations in the lateral intraparietal area. Journal of Neurophysiology, 76, 2071-2076.

Vuilleumier, P., Valenza, N., Mayer, E., Perrig, S., \& Landis, T. (1999). To see better when looking more to the right: Effects of gaze direction and frames of spatial coordinates in unilateral neglect. Journal of the International Neuropsychological Society, 5, 75-82.

Walker, R., Findlay, J. M., Young, A. W., \& Welch, J. (1991). Disentangling neglect and hemianopia. Neuropsychologia, 29, 1019-1027.

Wilson, B., Cockburn, J., \& Halligan, P. W. (1987). Behavioral inattention test. Suffolk, England: Thames Valley Test Company.

Xing, J., \& Andersen, R. A. (2000a). Memory activity of LIP neurons for sequential eye movements simulated with neural networks. Journal of Neurophysiology, 84, 651-665.

Xing, J., \& Andersen, R. A. (2000b). Models of the posterior parietal cortex which perform multimodal integration and represent space in several coordinate frames. Journal of Cognitive Neuroscience, 12, 601-614.

Young, L. R., \& Sheena, D. (1975). Survey of eye movement recording methods. Behavior Research Methods, Instruments and Computers, 7, 397-429.

Zihl, J. (1995). Visual scanning behavior in patients with homonymous hemianopia. Neuropsychologia, 33, 287-303.

Zipser, D., \& Andersen, R. A. (1988). A back-propagation programmed network that simulates response properties of a subset of posterior parietal neurons. Nature, 331, 679-684. 